\title{
Improved formalism for precision Higgs coupling fits
}

\author{
Tim Barklow, ${ }^{1}$ Keisuke Fujii, ${ }^{2}$ Sunghoon Jung, ${ }^{1,3}$ Robert Karl, ${ }^{4}$ Jenny List, ${ }^{4}$ \\ Tomohisa Ogawa, ${ }^{2}$ Michael E. Peskin, ${ }^{1}$ and Junping Tian ${ }^{5}$ \\ ${ }^{1}$ SLAC, Stanford University, Menlo Park, California 94025, USA \\ ${ }^{2}$ High Energy Accelerator Research Organization (KEK), Tsukuba, Ibaraki 305-0801, Japan \\ ${ }^{3}$ Department of Physics and Astronomy, Seoul National University, Seoul 08826, Korea \\ ${ }^{4}$ DESY, Notkestrasse 85, 22607 Hamburg, Germany \\ ${ }^{5}$ ICEPP, University of Tokyo, Hongo, Bunkyo-ku, Tokyo 113-0033, Japan
}

(Received 6 September 2017; published 20 March 2018)

\begin{abstract}
Future $e^{+} e^{-}$colliders give the promise of model-independent determinations of the couplings of the Higgs boson. In this paper, we present an improved formalism for extracting Higgs boson couplings from $e^{+} e^{-}$data, based on the effective field theory description of corrections to the Standard Model. We apply this formalism to give projections of Higgs coupling accuracies for stages of the International Linear Collider and for other proposed $e^{+} e^{-}$colliders.
\end{abstract}

DOI: 10.1103/PhysRevD.97.053003

\section{INTRODUCTION}

One of the most important opportunities provided by future $e^{+} e^{-}$colliders is that of determining the couplings of the Higgs boson with high precision and in a modelindependent way. By now, many studies have made projections of the accuracy with which Higgs couplings can be determined at proposed $e^{+} e^{-}$colliders [1-7]. Most of these studies are based on the $\kappa$ formalism, in which each Standard Model Higgs coupling is multiplied by an independent factor $\kappa_{I}$ and these factors are fit to expected measurements. The assumption is that introducing a large number of $\kappa_{I}$ parameters leads to coupling determinations with a great deal of model independence.

An alternative method, described in [8,9], takes a different point of view. This method begins from the assumption that the corrections to the Standard Model (SM) due to new physics can be parametrized by the addition of higher-dimension operators to the renormalizable (dimension-4) SM Lagrangian. We have come to realize that this approach is more correct in the way it takes into account the variety of effects that might arise from new physics. In addition, it allows us to incorporate powerful constraints from $S U(2) \times U(1)$ gauge invariance, and to make use of new observables that have not previously been considered in Higgs coupling fits. In this

Published by the American Physical Society under the terms of the Creative Commons Attribution 4.0 International license. Further distribution of this work must maintain attribution to the author(s) and the published article's title, journal citation, and DOI. Funded by SCOAP ${ }^{3}$. paper, we will formalize this approach, describe its advantages, and present projections of Higgs coupling accuracy for future $e^{+} e^{-}$colliders based on this formalism. Some recent analyses with similar ingredients but different emphases can be found in [10-13].

In particular, we make the assumption that the deviations from the SM predictions for the Higgs couplings can be represented by the addition of dimension- 6 operators. There are a large number of possible dimension- 6 operator coefficients - 84 in all — but only a manageable number of these play a role in the analysis of Higgs couplings. We will refer to the effective field theory (EFT) operator coefficients as $c_{J}$, to distinguish them from the $\kappa_{I}$.

The EFT approach is largely now adopted in the analysis of Higgs coupling data from the LHC; see [14]. However, the information on the EFT coefficients that will come from future $e^{+} e^{-}$colliders will be much more complete and specific. In fact, it is shown in some detail in the accompanying paper [15] that data from future $e^{+} e^{-}$ colliders can determine, independently and without ambiguity, all of the dimension-6 EFT coefficients that contribute directly to Higgs boson production and decay processes at those colliders. We can then use the EFT approach to provide estimates of Higgs boson couplings that are completely model-independent as long as the general framework of the EFT is valid.

Because one EFT operator can be exchanged for another by the use of the equations of motion, there are several different conventions used for the $c_{I}$. In this paper, we will use a variant of the "Warsaw basis" introduced in [16]. The notation, and detailed formulas for the linear deviations of the Higgs couplings, can be found in [15]. A similar set of formulas in the "SILH basis" $[8,9]$ can be found in $[13,17]$. 


\section{WHY IS $\kappa_{Z}$ MODEL DEPENDENT?}

Let us get right to the point: Why is a formalism based on $\kappa_{I}$ not model independent?

For Higgs decays to fermions, the corrections to the Standard Model are described phenomenologically by a single operator whose effect can be described by a $\kappa_{I}$ rescaling. The same is true for decays to $g g$ or $\gamma \gamma$. However, for the Higgs coupling to $W W$ and $Z Z$, this is not correct. The EFT actually leads to two distinct structures. We can represent the Higgs $Z$ interaction as parametrized by two coefficients $\eta_{Z}, \zeta_{Z}$,

$$
\delta \mathcal{L}=\left(1+\eta_{Z}\right) \frac{m_{Z}^{2}}{v} h Z_{\mu} Z^{\mu}+\zeta_{Z} \frac{h}{2 v} Z_{\mu \nu} Z^{\mu \nu},
$$

where $Z_{\mu \nu}$ is the $Z$ field strength. A similar formula can be written for the Higgs- $W$ interaction. The coefficients $\eta_{Z}, \eta_{W}$ multiply vertices with the same form as the SM vertices, but the $\zeta_{W}$ and $\zeta_{Z}$ terms bring in a new interactions of a different form. The $\eta$ and $\zeta$ parameters are derived from the EFT operator coefficients $c_{I}$ in a way that we will discuss in a moment.

The $\zeta$ terms involve the field strengths of the vector fields, and so are momentum dependent. The effect of these terms depends on the momenta of the two vector bosons and the extent to which these are off shell. For a $125 \mathrm{GeV}$ Higgs boson and the cross section at $250 \mathrm{GeV}$ in the center of mass, we find, to linear order in the corrections,

$$
\begin{aligned}
\sigma\left(e^{+} e^{-} \rightarrow Z h\right) & =(S M) \cdot\left(1+2 \eta_{Z}+(5.7) \zeta_{Z}\right) \\
\Gamma\left(h \rightarrow W W^{*}\right) & =(S M) \cdot\left(1+2 \eta_{W}-(0.78) \zeta_{W}\right) \\
\Gamma\left(h \rightarrow Z Z^{*}\right) & =(S M) \cdot\left(1+2 \eta_{Z}-(0.50) \zeta_{Z}\right) .
\end{aligned}
$$

The coefficients in front of the $\zeta$ terms come from integrals over the relevant phase space for each process.

In weakly coupled extensions of the Higgs sector, including supersymmetry, the $\zeta$ coefficients typically arise from loop diagrams and have values of $10^{-3}$ or smaller, but in Little Higgs and Randall-Sundrum models (without T-parity), these coefficients can be present at the tree level and can be as large as other new physics contributions [8,18]. A simple $\kappa_{I}$ parametrization cannot incorporate this degree of freedom. Thus, we conclude, the $\kappa_{I}$ formalism is not model independent and cannot provide a general basis for tests of models of new physics effects on the Higgs couplings against data.

The EFT analysis adds parameters to the standard $\kappa_{I}$ scheme, but it also has a compensatory advantage. New physics corrections can modify the relative size of the Higgs boson couplings to $Z$ and $W$. In the usual modelindependent $\kappa_{I}$ analysis, this is accounted by taking $\kappa_{Z}$ and $\kappa_{W}$ to be independent parameters that can vary arbitrarily with respect to one another. In the EFT approach, the largest contributions to the parameters $\eta_{Z}$ and $\eta_{W}$ and to $\zeta_{Z}$ and $\zeta_{W}$ come from the same dimension- 6 operator coefficients. More explicitly, we find

$$
\eta_{W}=-\frac{1}{2} c_{H} \quad \eta_{Z}=-\frac{1}{2} c_{H}-c_{T},
$$

where $c_{T}$ is related to the $T$ parameter of precision electroweak analysis [19] and is constrained by that analysis to be very small. The parameter $c_{H}$ is an overall renormalization of the Higgs field as discussed in [8,20,21]. Similarly, with $\left(c_{w}, s_{w}\right)=\left(\cos \theta_{w}, \sin \theta_{w}\right)$, we find

$$
\begin{aligned}
& \zeta_{W}=\left(8 c_{W W}\right) \\
& \zeta_{Z}=c_{w}^{2}\left(8 c_{W W}\right)+2 s_{w}^{2}\left(8 c_{W B}\right)+\left(s_{w}^{4} / c_{w}^{2}\right)\left(8 c_{B B}\right),
\end{aligned}
$$

where $c_{W W}, c_{W B}$, and $c_{B B}$ are coefficients of dimension-6 operators with the squares of $S U(2) \times U(1)$ field strengths. The parameters $c_{W B}$ and $c_{B B}$ are strongly constrained by measurements outside the program of $e^{+} e^{-}$measurements of Higgs reactions. Thus, in the EFT approach, the relative sizes of the two $Z$ and $W$ couplings are regulated by $S U(2) \times U(1)$ gauge invariance in a way that their relation can be determined from data. The overall effect is that we exchange the two parameters $\kappa_{Z}, \kappa_{W}$ for two parameters $\eta_{Z}$, $\zeta_{Z}$, with no new freedom for $\eta_{W}$ and $\zeta_{W}$.

The structure of the Higgs couplings to $W$ and $Z$ is important to resolve the trickiest and most subtle problem of Higgs coupling analysis. Experiments measure branching ratios, but models of new physics predict the absolute strengths of Higgs couplings and, through these, the Higgs partial widths. To effectively compare theory and experiment, it is necessary to find the absolute normalization of the partial widths. The conversion factor is the Higgs boson total width. This width, about $4 \mathrm{MeV}$ in the SM, is too small to be measured directly at any proposed accelerator. Rather, it must be extracted from the fit to coupling constants.

In the literature, this is typically done within the $\kappa$ framework by assuming that the total cross section for $e^{+} e^{-} \rightarrow Z h$ and the partial width $\Gamma\left(h \rightarrow Z Z^{*}\right)$ are both proportional to the parameter $\kappa_{Z}$. This total cross section can be measured by observing the recoil $Z$ at a fixed lab energy, independently of the Higgs decay scheme. This determines $\kappa_{Z}$ to high accuracy. The Higgs width can then be extracted from the ratio of measurable quantities,

$$
\frac{\sigma\left(e^{+} e^{-} \rightarrow Z h\right)}{\operatorname{BR}\left(h \rightarrow Z Z^{*}\right)}=\frac{\sigma\left(e^{+} e^{-} \rightarrow Z h\right)}{\Gamma\left(h \rightarrow Z Z^{*}\right) / \Gamma_{h}} \sim \Gamma_{h},
$$

from which $\kappa_{Z}$ cancels out. Since $\operatorname{BR}\left(h \rightarrow Z Z^{*}\right)$ is small, about $3 \%$ in the SM, this determination of $\Gamma_{h}$ suffers from low statistics, but, at least, it seems to be model independent.

The presence of the $h Z Z$ coupling proportional to $\zeta_{Z}$ ruins this strategy. We see from (2) that the numerator and denominator of (5) have completely different dependence on $\zeta_{Z}$, even with a different sign. To overcome this problem, we need a separate method to determine the size of the $\zeta_{Z}$ terms. We will discuss this in the next section. 


\section{ELEMENTS OF A FIT FOR $\eta_{Z}$ AND $\xi_{Z}$}

There are a number of possible methods to determine the size of the $\zeta$ parameters. In this section, we will highlight one particularly powerful method, which is to make use of the angular distribution and polarization asymmetries of the reaction $e^{+} e^{-} \rightarrow Z h$. These observables have not previously been applied to Higgs coupling analysis.

The contributions to the $e^{+} e^{-} \rightarrow Z h$ cross section from the $\eta_{Z}$ and $\zeta_{Z}$ terms can be distinguished by their effects on these angular distributions and asymmetries. The $\eta_{Z}$ terms lead to enhanced amplitudes for longitudinal $Z$ polarization and to production at smaller values of $|\cos \theta|$, while the $\zeta_{Z}$ terms lead to equal production of the three $Z$ polarization states at higher values of $|\cos \theta|$. At $250 \mathrm{GeV}$, this is a relatively small effect, proportional to $\left(E_{Z}^{2} / m_{Z}^{2}-1\right)=0.47$, but it becomes larger at higher energy. Second, the contribution from the $\zeta_{Z}$ term is quite sensitive to beam polarization. Beam polarization is straightforward to achieve at linear colliders but is not projected for circular colliders, while circular collider designs have higher luminosity at $250 \mathrm{GeV}$. Then there is a certain complementarity between these approaches.

The polarization effect in the $e^{+} e^{-} \rightarrow Z h$ cross section arises from the interference of $s$-channel diagrams with $Z$ and $A$; see Fig. 1. In addition to the $\zeta_{Z}$ term in the Higgs Lagrangian, the dimension- 6 operators in the EFT induce a term

$$
\delta \mathcal{L}=\zeta_{A Z} \frac{h}{v} A_{\mu \nu} Z^{\mu \nu}
$$

that mixes the $A$ and $Z$ field strengths. The coefficient of this term is related to the parameters already discussed by

$\zeta_{A Z}=s_{w} c_{w}\left(8 c_{W W}\right)-\frac{s_{w}}{c_{w}}\left(c_{w}^{2}-s_{w}^{2}\right)\left(8 c_{W B}\right)-\frac{s_{w}^{3}}{c_{w}}\left(8 c_{B B}\right)$.

This produces the second diagram in Fig. 1, which is not present at tree level in the SM. There is also a third contribution that has the form of a contact interaction. This third diagram is parametrically enhanced by terms of order $s / m_{Z}^{2}$ [22]. However, the EFT coefficients that appear in this diagram $\left(c_{H L}, c_{H L}^{\prime}, c_{H E}\right)$ are very strongly constrained by the analysis of precision electroweak corrections and $e^{+} e^{-} \rightarrow W^{+} W^{-}$, in a way that compensates this

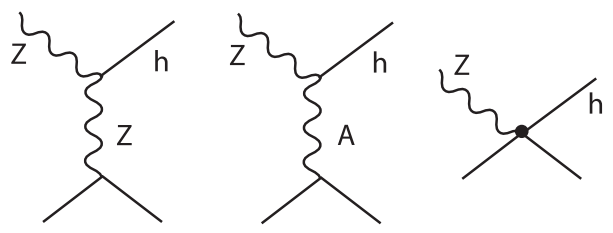

FIG. 1. Diagrams contributing to the cross section for $e^{+} e^{-} \rightarrow$ $Z h$ in the EFT description of Higgs couplings. The second and third diagrams are not present in the Standard Model. They arise from the dimension-6 operators in the EFT. enhancement [15]. We will ignore this diagram in the simplified analysis presented in this section. However, it does play a role in the more complete analysis that we will describe in Sec. IV.

The sign of the interference term between the $s$-channel $Z$ and $A$ diagrams depends on the beam polarization. The $Z$ charge of the electron changes sign between $e_{L}^{-}$and $e_{R}^{-}$, $\left(\frac{1}{2}-s_{w}\right)^{2} \rightarrow\left(-s_{w}^{2}\right)$, while the $A$ charge stays the same. This leads to a near cancellation of the $\zeta_{Z}$ terms for $e_{R}^{-}$, while there is constructive interference for $e_{L}^{-}$.

To illustrate these effects, we carry out a simplified fit to the Higgs couplings in the following framework: The starting point is the table of projected errors on the total $e^{+} e^{-} \rightarrow Z h$ cross section given in Appendix A. These error estimates are based on full simulation studies with the ILD detector model used for ILC studies [23,24]. The estimates are provided for each of two configurations of beam polarization, an $L$ beam with electron and positron beam polarizations $(-0.8,+0.3)$ and an $R$ beam with electron and positron beam polarizations $(+0.8,-0.3)$. These errors are essentially identical for the two beam polarizations, and so we can apply them also for unpolarized beams. All of the analyses below are carried out at the linearized level.

A fit in the $\kappa$ framework would modify each Higgs couplings by

$$
g_{h A \bar{A}}=g_{h A \bar{A}}\left(1+\delta \kappa_{A}\right) .
$$

For reference, we carried out a $\kappa$ fit to this data from $e^{+} e^{-} \rightarrow Z h$ with seven parameters:

$$
\delta \kappa_{Z}, \delta \kappa_{W}, \delta \kappa_{b}, \delta \kappa_{c}, \delta \kappa_{g}, \delta \kappa_{\tau}, \delta \kappa_{\mu}
$$

In addition, we allow branching ratios of the Higgs boson to invisible and to noninvisible exotic decay modes. This modification of the Standard Model is usually omitted in EFT fits, which concentrate on the effects of heavy particles. However, the search for Higgs decays to light invisible and exotic particles is an important part of the full $e^{+} e^{-}$program, and the possibility of such decays adds an uncertainty to the extraction of the Higgs boson total width that should be accounted. To parametrize these possible exotic Higgs decays, we introduce two additional parameters $\delta a_{\text {inv }}$ and $\delta a_{\text {other }}$. The parameter $\delta a_{\text {inv }}$ is the fraction of all Higgs decays that go to completely invisible decay products,

$$
\delta a_{\mathrm{inv}}=\Gamma(h \rightarrow \mathrm{invis}) / \Gamma_{h, S M} .
$$

Similarly, $\delta a_{\text {other }}$ is the fraction of all Higgs decays that do not fit into any standard category, or even into the category of invisible decays. It is extremely conservative to include the $\delta a_{\text {other }}$ parameter, since, at an $e^{+} e^{-}$collider, almost any exotic decay will be observed and recognized as such [25]. But this is the way that all previous "model-independent" Higgs coupling fits for $e^{+} e^{-}$colliders have been done. 
The simplified fits in the EFT framework also uses nine parameters. These are the EFT parameters $c_{H}$ and $c_{W W}$, the EFT parameters that shift the Higgs couplings $b, c, g, \tau$, and $\mu$, and the $a_{\text {inv }}$ and $a_{\text {other }}$ parameters described above (10) [26]. In this simplified fit, $c_{W B}$ and $c_{B B}$ are set equal to zero. In the complete fit described below, these latter parameters are determined by constraints from precision electroweak measurements, $e^{+} e^{-} \rightarrow W^{+} W^{-}$, and $\Gamma(h \rightarrow \gamma \gamma)$.

The full details of our treatment of the $e^{+} e^{-} \rightarrow Z h$ cross section and angular distribution are discussed in [15]. Our approach can be summarized by saying that the perturbations to the SM cross section from the $\eta_{Z}$ and $\zeta_{Z}$ terms can be completely parametrized in terms of coefficients $a$ and $b$, respectively, which describe the variations in the cross section, angular distribution, and $Z$ polarization. These $a$ and $b$ parameters depend on beam polarization and center of mass energy. For example, at the tree level, the total cross section for $e^{+} e^{-} \rightarrow Z h$ from a fully polarized $e_{L}^{-} e_{R}^{+}$or $e_{R}^{-} e_{L}^{+}$initial state is given by

$$
\begin{aligned}
& \sigma= \frac{2}{3} \frac{\pi \alpha_{w}^{2}}{c_{w}^{4}} \frac{m_{Z}^{2}}{\left(s-m_{Z}^{2}\right)} \frac{2 k_{Z}}{\sqrt{s}}\left(2+\frac{E_{Z}^{2}}{m_{Z}^{2}}\right) \cdot Q_{Z}^{2} \\
& \cdot\left[1+2 a+2 \frac{3 \sqrt{s} E_{Z} / m_{Z}^{2}}{\left(2+E_{Z}^{2} / m_{Z}^{2}\right)} b\right],
\end{aligned}
$$

where $k_{Z}, E_{Z}$ are the lab frame momentum and energy of the $Z$. In the simplified parameter set used here, the parameters in (11) are given, for a fully polarized $e_{L}^{-} e_{R}^{+}$ initial state, by

$$
\begin{aligned}
Q_{Z L} & =\left(\frac{1}{2}-s_{w}^{2}\right), \quad a_{L}=-c_{H} / 2 \\
b_{L} & =c_{w}^{2}\left(1+\frac{s_{w}^{2}}{1 / 2-s_{w}^{2}} \frac{s-m_{Z}^{2}}{s}\right)\left(8 c_{W W}\right)
\end{aligned}
$$

and, for a fully polarized $e_{R}^{-} e_{L}^{+}$initial state, by

$$
\begin{aligned}
Q_{Z R} & =\left(-s_{w}^{2}\right), \quad a_{R}=-c_{H} / 2 \\
b_{R} & =c_{w}^{2}\left(1-\frac{s-m_{Z}^{2}}{s}\right)\left(8 c_{W W}\right) .
\end{aligned}
$$

The change of sign between the two terms in $b_{L}$ vs $b_{R}$ is the polarization effect described earlier in this section. There are similar formulas for the distributions in production angle and $Z$ decay angles; see [15] for details. Fits for the $a$ and $b$ parameters using ILD full simulation data are described in [24], and these are the basis for the error estimates and correlations for these parameters listed in Appendix A.

The results of the simplified fits are shown in Table I. We assume $2000 \mathrm{fb}^{-1}$ of data, equally divided between the two polarized beam configurations. These fits include only data from $e^{+} e^{-} \rightarrow Z h$. The $\kappa$ fit is limited by the poor knowledge of the Higgs total width. In this fit, the width is obtained through the relation (5) and suffers from a lack of statistics for the $h \rightarrow Z Z^{*}$ decay. In the EFT fits, the
TABLE I. Projected relative errors for Higgs boson couplings and other Higgs observables, in percent, for the fits described in Sec. III. The first column gives results of a fit with simple $\kappa$ rescaling of the Standard Model Higgs couplings. The next three columns give results of fits to the EFT coefficients using the simplified nineparameter framework described in Sec. III. The second column uses only angular information for the process $e^{+} e^{-} \rightarrow Z h$; the third column uses only the polarized beam total cross sections for this reaction; the fourth column uses both sets of information. The final column gives the result of the full EFT fit described in Sec. IV. All of these fits assume data samples of $2 \mathrm{ab}^{-1}$ at $250 \mathrm{GeV}$. The effective couplings $g(h W W)$ and $g(h Z Z)$ are defined as proportional to the square root of the corresponding partial widths. The last two lines give $95 \%$ confidence upper limits on the exotic branching ratios.

\begin{tabular}{lccccc}
\hline \hline & $\begin{array}{c}\kappa \\
\text { fit }\end{array}$ & $\begin{array}{c}\text { Angl. } \\
\text { only }\end{array}$ & $\begin{array}{c}\text { Pol. } \\
\text { only }\end{array}$ & Both & $\begin{array}{c}\text { Full EFT } \\
\text { fit }\end{array}$ \\
\hline$g(h b \bar{b})$ & 3.21 & 3.87 & 0.94 & 0.94 & 1.04 \\
$g(h c \bar{c})$ & 3.52 & 4.19 & 1.73 & 1.73 & 1.79 \\
$g(h g g)$ & 3.43 & 4.10 & 1.54 & 1.54 & 1.60 \\
$g(h W W)$ & 3.31 & 3.77 & 0.46 & 0.45 & 0.65 \\
$g(h \tau \tau)$ & 3.25 & 3.91 & 1.07 & 1.07 & 1.16 \\
$g(h Z Z)$ & 0.36 & 3.51 & 0.45 & 0.44 & 0.66 \\
$g(h \mu \mu)$ & 13.1 & 14.7 & 12.8 & 12.8 & 5.53 \\
$g(h b \bar{b}) / g(h W W)$ & 0.85 & 0.92 & 0.83 & 0.83 & 0.82 \\
$g(h W W) / g(h Z Z)$ & 3.29 & 0.26 & 0.02 & 0.02 & 0.07 \\
$\Gamma_{h}$ & 6.53 & 7.64 & 2.20 & 2.18 & 2.38 \\
$\sigma\left(e^{+} e^{-} \rightarrow Z h\right)$ & 0.72 & 0.80 & 0.72 & 0.70 & 0.70 \\
$\mathrm{BR}(h \rightarrow$ inv $)$ & 0.39 & 0.36 & 0.30 & 0.30 & 0.30 \\
$\mathrm{BR}(h \rightarrow$ other $)$ & 1.57 & 1.71 & 1.53 & 1.51 & 1.50 \\
\hline \hline
\end{tabular}

uncertainty in the couplings reflects the uncertainty in the knowledge of the $\zeta_{Z}$ parameter, which is determined mainly from the data on the reactions $e^{+} e^{-} \rightarrow Z h$. Note that polarization is in general a more powerful analyzer for $\zeta_{Z}$ than the angular distributions, although either method can be effective with a sufficiently large luminosity sample. For reference, the last column of the fit gives the results of the full EFT fit described in the next section. The simplified fit is quite idealized, but its outcome turns out to be close to that of a full EFT analysis.

\section{PROJECTIONS FOR ILC AT 250 GEV}

A complete analysis within the EFT framework requires a much larger number of parameters. As stated above, there are 84 possible dimension-6 operators with the gauge symmetry and particle content of the Standard Model. In [15], we point to a subset of nine of these operators that contribute to processes involving $W, Z$, Higgs, and light leptons only. Five additional operators modify the Higgs couplings to fermions and gluons. Two further parameters are needed to describe the $W$ and $Z$ couplings to quarks. Then our model-independent fit will involve 16 dimension- 6 operator coefficients plus the four relevant parameters of the 
Standard Model and the two parameters introduced above for exotic Higgs decays. The total number of parameters is 22 . Though many papers have been written about fits to Higgs data using EFT, it seems not to have been realized that data from future $e^{+} e^{-}$colliders will completely constraint these 22 parameters, allowing precise analyses that are model independent to the extent that this subset of operators gives a general description of new physics.

The subset of nine operators noted above does not include the most general operators with $W, Z$, Higgs, and light leptons. It excludes 4-fermion contact interactions, which, however, do not contribute to the observables of relevance here. It assumes muon-electron universality, which can be strongly tested at $e^{+} e^{-}$colliders in $W$ decays and in 2fermion scattering processes. It excludes $C P$ violating operators. But $C P$ violating operators contribute only in order $c_{I}^{2}$ to the observables we consider, and there are other observables linear in these $c_{I}$ (for example, the forwardbackward asymmetry in $e^{+} e^{-} \rightarrow Z h$ ) that can bound them at the percent level. It excludes the coefficient $c_{6}$ that shifts the triple-Higgs coupling, which does not contribute at tree level to the observables we consider here [27]. Some additional qualifications are given Sec. II B of [15]. Most importantly, our analysis assumes that operators of dimension- 8 are negligible and operators of dimension- 6 can be treated in linear order only. This makes sense for operators that provide few-percent corrections to the Higgs couplings, corresponding to the sensitivity of future $e^{+} e^{-}$colliders. In this paper, we will treat the dimension -6 operators at the tree level only. If corrections to Higgs couplings turn out to be at the $30 \%$ level, one might question this assumption. But unless corrections to the Higgs couplings are very large, our restricted-but still 22-dimensional-parameter set can be considered a model-independent description of new physics for the purpose of Higgs coupling analysis.

The fit parameters are the following: First, since dimension-6 effects renormalize the parameters of the Standard Model, we must include deviations in the four Standard Model parameters,

$$
\delta g, \delta g^{\prime}, \delta v, \delta \lambda
$$

In the basis chosen in [15], the nine EFT parameters for the Higgs and electroweak boson sector are

$$
c_{T}, c_{W W}, c_{W B}, c_{B B}, c_{3 W}, c_{H L}, c_{H L}^{\prime}, c_{H E}, c_{H} .
$$

Of these, the parameter $c_{T}$ is essentially the $T$ parameter of precision electroweak analysis. The parameters $c_{H L}, c_{H L}^{\prime}$, $c_{H E}$ parametrize current-current interactions between the Higgs boson and the leptons. The parameters $c_{W W}, c_{W B}$, and $c_{B B}$ parametrize operators quadratic in vector boson field strengths, and $c_{3 W}$ parametrizes the one possible operator cubic in the $W$ field strength. We need five additional coefficients to describe the shifts in the Higgs couplings to fermions and gluons [26],

$$
c_{H b}, c_{H c}, c_{H \tau}, c_{H \mu}, c_{H g} .
$$

For our treatment of the Higgs decays to $W W^{*}$ and $Z Z^{*}$, we need two further combinations of EFT coefficients, called $C_{W}$ and $C_{Z}$ in [15], which are measurable from the $W$ and $Z$ boson total widths. Finally, we include the two parameters $\delta a_{\text {inv }}$ and $\delta a_{\text {other }}$ introduced above (10).

Given this parameter set, we assume a linear relation between the parameters and observables,

$$
\mathcal{O}_{i}=\mathcal{O}_{i, S M}+V_{i J} c_{J}
$$

Then measurements of the $\mathcal{O}_{i}$ lead to a covariance matrix for the $c_{J}$, which can then be translated into projected errors on Higgs partial widths or Higgs couplings.

As inputs to this process, we take the following information: First, seven quantities very well measured in precision electroweak studies- $\alpha, G_{F}, m_{W}, m_{Z}, A_{\ell}$, and $\Gamma\left(Z \rightarrow \ell^{+} \ell^{-}\right)$-provide seven strong constraints on the parameter set. Note that we do not need to make any assumption that the electroweak corrections are "oblique" in the sense of [19]. For the $h \rightarrow W W^{*}$ and $h \rightarrow Z Z^{*}$ partial widths, we also must input the measured values of the $W$ and $Z$ total widths, as described in [15]. At the level of dimension-6 operators, $e^{+} e^{-} \rightarrow W^{+} W^{-}$provides three independent new physics parameters- $g_{1 Z}, \kappa_{A}$, and $\lambda_{A}-$ that can be constrained by measurement. LEP and LHC measurements already constrain these parameters at the $1 \%$ level. For this analysis, we need more than an order of magnitude improvement in the constraints, but we expect that this will be provided by $e^{+} e^{-}$colliders at the same time that they measure the Higgs boson parameters [28]. A projected covariance matrix for these parameters with $2000 \mathrm{fb}^{-1}$ of data at $250 \mathrm{GeV}$, estimated from ILD studies of $e^{+} e^{-} \rightarrow W^{+} W^{-}$at higher energies by Marchesini [29] and Rosca [30], is given in Appendix A. The LHC experiments will provide a strong measurement of the ratio $\mathrm{BR}\left(h \rightarrow Z Z^{*}\right) / \mathrm{BR}(h \rightarrow \gamma \gamma)$. The ATLAS analysis [31] estimates the error on this measurement after $3000 \mathrm{fb}^{-1}$ of data taking as $3.6 \%-4 \%$. We expect that a measurement strategy dedicated to canceling systematic errors between these two similar and characteristic processes can reach the statistics limit of $2 \%$. We use this latter number in our fit, but the results are not changed if the error is indeed $4 \%$. This provides the strongest constraint on $c_{B B}$. The expected LHC measurement of $\operatorname{BR}(h \rightarrow Z \gamma) / \mathrm{BR}(h \rightarrow \gamma \gamma)$, with an error of $31 \%$ [32], also provides a significant constraint on $c_{W B}$. We also input the expected LHC measurement of $\operatorname{BR}\left(h \rightarrow \mu^{+} \mu^{-}\right) / \operatorname{BR}(h \rightarrow \gamma \gamma)$ [31]. The input values used are listed in Appendix A.

At this stage, the measurements described constrain 13 of the original 22 parameters. This includes all but two of the $4+9$ parameters relevant to $W, Z$, Higgs, and light lepton processes. The remaining two free parameters are $c_{H}$ and $c_{W W}$, the parameters of the simplified fit described in 
the previous section. These parameters are constrained by measurements of the total cross section, angular distribution, and polarization asymmetries in $e^{+} e^{-} \rightarrow Z h$, as explained in the previous section.

The remaining nine parameters are those that appear only in expressions for the Higgs boson partial widths. To include these parameters, we add measurements of $\sigma$. $B R$ for $e^{+} e^{-} \rightarrow Z h$ followed by Higgs boson decay to specific final states. The decay widths to $W W^{*}$ and $Z Z^{*}$ also depend on $c_{H}$ and $c_{W W}$ in a way that put additional constraints on these parameters. This makes the fit for these parameters more robust and decreases its dependence on any particular input.

Though Higgs production through $W$ fusion has a small cross section at $250 \mathrm{GeV}$, we include the measurement of the rate for $e^{+} e^{-} \rightarrow \nu \bar{\nu} h, h \rightarrow b \bar{b}$.

The full fit contains a number of mechanisms for constraining the $\zeta_{W}$ and $\zeta_{Z}$ parameters, or, alternatively through (4), the EFT coefficients $c_{W W}, c_{W B}$, and $c_{B B}$. We have explained in Sec. III how these parameters are constrained by measurements of $e^{+} e^{-} \rightarrow Z h$. The partial widths for Higgs decay to $W W^{*}$ and $Z Z^{*}$ also contain these parameters, so measurements of Higgs processes with vector bosons in the final state give constraints. The coefficient $c_{W B}$ is directly constrained by measurements of $e^{+} e^{-} \rightarrow W^{+} W^{-}$angular distributions. Linear combinations of the three coefficients give new tree-level contributions to the Higgs decay widths to $\gamma \gamma$ and $Z \gamma$, so that the LHC measurements of ratios of branching ratios provide constraints. The effect of the various inputs in determining these coefficients is shown as a systematic progression in Table II of [15]. The fact that several different inputs contribute decreases the importance of beam polarization for achieving accurate determinations of the Higgs boson couplings with respect to the results of the simple fits described in Sec. III.

On the other hand, there is another effect that must also be accounted. The EFT formalism leads to new contact interactions, of which an example is the third diagram in Fig. 1 [22]. As noted above, these diagrams are enhanced by a factor $s / m_{Z}^{2}$. They are strongly constrained only when the full set of Higgs processes measurable at $e^{+} e^{-}$colliders is included. The influence of these diagrams, and the role of the inputs in controlling them, is described in some detail in Sec. V of [15].

The results of the full 22-parameter fit are shown in the last column of Table I. The results are quite similar to those from the simple fit of the previous section. The introduction of many new parameters does not decrease the quality of the fit, since the additional measurements constrain these parameters strongly. The largest effect is seen in the $h W W$ and $h Z Z$ couplings, where a contribution from $c_{W B}$ adds a small amount to the total error. The full fit also depends much less strongly on beam polarization, since this is now only one of several constraints that determine the parameter
TABLE II. Projected relative errors for Higgs boson couplings and other Higgs observables, in percent, from the EFT fits in this paper, compared to the results of Higgs coupling fits shown in Table I of [3]. The first column gives the result of the fit described in Sec. IV, with $2 \mathrm{ab}^{-1}$ of data at $250 \mathrm{GeV}$. The fourth column gives the results of Sec. VI, adding $4 \mathrm{ab}^{-1}$ at $500 \mathrm{GeV}$. The total data samples assumed in the third and fourth columns are the same.

\begin{tabular}{lcccc}
\hline \hline & $\begin{array}{c}\text { Full 250 GeV } \\
\text { EFT fit }\end{array}$ & $\begin{array}{c}\text { Initial } \\
\text { ILC [3] }\end{array}$ & $\begin{array}{c}\text { Full } \\
\text { ILC [3] }\end{array}$ & $\begin{array}{c}\text { Full ILC } \\
\text { EFT fit }\end{array}$ \\
\hline$g(h b \bar{b})$ & 1.04 & 1.5 & 0.7 & 0.55 \\
$g(h c \bar{c})$ & 1.79 & 2.7 & 1.2 & 1.09 \\
$g(h g g)$ & 1.60 & 2.3 & 1.0 & 0.89 \\
$g(h W W)$ & 0.65 & 0.81 & 0.42 & 0.34 \\
$g(h \tau \tau)$ & 1.16 & 1.9 & 0.9 & 0.71 \\
$g(h Z Z)$ & 0.66 & 0.58 & 0.31 & 0.34 \\
$g(h \mu \mu)$ & 5.53 & 20 & 9.2 & 4.95 \\
$\Gamma_{h}$ & 2.38 & 3.8 & 1.8 & 1.50 \\
\hline \hline
\end{tabular}

$c_{W W}$. We will see this in the examples presented in the next section.

Table II gives a comparison of this EFT fit to previous results that we have presented in the past for ILC, using the $\kappa$ framework but including measurements at $500 \mathrm{GeV}$ to sharpen the determination of the Higgs total width. The first column shows the result of this fit. The second and third columns give the results quoted in [3] for the initial and full phases of the ILC program described in [33]. The fourth column shows the results of the fit described in Sec. VI of this paper. It is interesting that, with the new analysis method that we present here, a long run at $250 \mathrm{GeV}$ gives considerable power for learning about the Higgs couplings even before we go to higher energy. Eventually, of course, we must do both. Running at $500 \mathrm{GeV}$ and above is also needed to complete the program of precision Higgs measurements by measuring the $h t \bar{t}$ coupling and the triple Higgs coupling.

\section{POLARIZATION VS LUMINOSITY VS ENERGY}

Beam polarization played an important role in the fits described in Secs. III and IV. However, as we pointed out in Sec. IV, inclusion of the full set of observables that can be measured at $e^{+} e^{-}$colliders takes some pressure off the requirement for beam polarization. Designs for the proposed circular $e^{+} e^{-}$colliders CEPC and FCC-ee anticipate larger event samples than ILC at $250 \mathrm{GeV}$, but do not anticipate longitudinally polarized beams. The proposed CLIC linear collider anticipates its initial run at a higher energy of $380 \mathrm{GeV}$. It is interesting to explore the trade-offs between these proposed programs. 
This question can be answered within the EFT formalism by using the data in Appendix A to estimate the inputs for the various accelerator schemes, and then performing a fits analogous to that of Sec. IV. The results are shown in Table III. The first column again shows the fit of Sec. IV, with polarized beams and $2 \mathrm{ab}^{-1}$ of integrated luminosity. The second column shows the result for the same integrated luminosity at $350 \mathrm{GeV}$ in the center of mass, appropriate for the proposed CLIC linear collider. (The CLIC proposal now considers running at $380 \mathrm{GeV}$, but all published studies have been done assuming $350 \mathrm{GeV}$. The CLIC proposal includes additional stages at higher energy that are not considered here $[6,34]$.) The third and fourth columns show the results for unpolarized beams [35] using the luminosity samples of $5 \mathrm{ab}^{-1}$, projected for CEPC [5], and for $5 \mathrm{ab}^{-1}$ at $250 \mathrm{GeV}$ plus $1.5 \mathrm{ab}^{-1}$ at $350 \mathrm{GeV}$, approximating the program projected for FCC-ee with two detectors [36]. Our error estimates include an accounting for expected systematic errors, as described in Appendix A.

The fifth column shows the results of a fit including data at $500 \mathrm{GeV}$ that will be described in the next section. This fit includes $2 \mathrm{ab}^{-1}$ at $250 \mathrm{GeV}$ plus $4 \mathrm{ab}^{-1}$ at $500 \mathrm{GeV}$, realizing the full ILC plan set out in [33].

It is clear from the table that the decreased power of the angular distributions to measure the $\zeta$ parameters can be compensated by higher luminosity. One should also remember that the ratios of branching ratios are measured at $e^{+} e^{-}$colliders without ambiguity, and the accuracy of these measurements improves as $\sqrt{N}$. These ratios of branching ratios can be important in the testing of specific models, as we will discuss in Sec. VII.

One should note that beam polarization offers some qualitative advantages that are not captured in a table such as this. Having separate samples with different beam polarization essentially doubles the number of independent observables and allows consistency tests that would not otherwise be available.

It is amusing to use comparisons such as this one to try to determine the "best" future $e^{+} e^{-}$collider, but truly the best collider is the one that is actually built. The trade-off shown between linear and circular colliders shows that colliders of both types have powerful capability for discovering new physics beyond the Standard Model. We will present an explicit comparison with models for ILC in Sec. VII. Similar results would be obtained with any of the scenarios shown in Table III.

It is of some interest to understand the importance of positron polarization, in addition to electron polarization, since positron polarization at linear colliders requires a special type of positron source. Table IV investigates this question by comparing the results of a complete EFT fit at $250 \mathrm{GeV}$ and $2000 \mathrm{fb}^{-1}$ for different assumptions about the electron and positron polarization.

TABLE III. Projected relative errors for Higgs boson couplings and other Higgs observables, in percent, comparing the full EFT fit described in Sec. IV to other possible $e^{+} e^{-}$collider scenarios. The first column shows the results of the fit at $250 \mathrm{GeV}$ with $2 \mathrm{ab}^{-1}$ of data, $80 \%$ electron polarization, and $30 \%$ positron polarization, as expected in the ILC design. The second column shows a fit at $350 \mathrm{GeV}$ with $2 \mathrm{ab}^{-1}$ of data, $80 \%$ electron polarization, and no positron polarization. The third and fourth columns show scenarios with no electron or positron polarization but higher integrated luminosity, $5 \mathrm{ab}^{-1}$ at $250 \mathrm{GeV}$ in the third column and $5 \mathrm{ab}^{-1}$ at $250 \mathrm{GeV}$ plus $1.5 \mathrm{ab}^{-1}$ at $350 \mathrm{GeV}$ in the fourth column. The fifth column gives the result of the fit described in Sec. VI including data from 250 and $500 \mathrm{GeV}$. The notation is as in Table I.

\begin{tabular}{lccccc}
\hline \hline & $\begin{array}{c}2 \mathrm{ab}^{-1} \\
\text { with pol. }\end{array}$ & $\begin{array}{c}2 \mathrm{ab}^{-1} \\
350 \mathrm{GeV}\end{array}$ & $\begin{array}{c}5 \mathrm{ab}^{-1} \\
\text { No pol. }\end{array}$ & $\begin{array}{c}\text { At 350 GeV } \\
\text { At } \mathrm{ab}^{-1}\end{array}$ & $\begin{array}{c}\text { Full ILC } \\
250+500 \mathrm{GeV}\end{array}$ \\
\hline$g(h b \bar{b})$ & 1.04 & 1.08 & 0.98 & 0.66 & 0.55 \\
$g(h c \bar{c})$ & 1.79 & 2.27 & 1.42 & 1.15 & 1.09 \\
$g(h g g)$ & 1.60 & 1.65 & 1.31 & 0.99 & 0.89 \\
$g(h W W)$ & 0.65 & 0.56 & 0.80 & 0.42 & 0.34 \\
$g(h \tau \tau)$ & 1.16 & 1.35 & 1.06 & 0.75 & 0.71 \\
$g(h Z Z)$ & 0.66 & 0.57 & 0.80 & 0.42 & 0.34 \\
$g(h \gamma \gamma)$ & 1.20 & 1.15 & 1.26 & 1.04 & 1.01 \\
$g(h \mu \mu)$ & 5.53 & 5.71 & 5.10 & 4.87 & 4.95 \\
$g(h b b) / g(h W W)$ & 0.82 & 0.90 & 0.58 & 0.51 & 0.43 \\
$g(h W W) / g(h Z Z)$ & 0.07 & 0.06 & 0.07 & 0.06 & 0.05 \\
$\Gamma_{h}$ & 2.38 & 2.50 & 2.11 & 1.49 & 1.50 \\
$\sigma\left(e^{+} e^{-} \rightarrow Z h\right)$ & 0.70 & 0.77 & 0.50 & 0.22 & 0.61 \\
$\mathrm{BR}(h \rightarrow$ inv $)$ & 0.30 & 0.56 & 0.30 & 0.27 & 0.28 \\
$\mathrm{BR}(h \rightarrow$ other $)$ & 1.50 & 1.63 & 1.09 & 0.94 & 1.15 \\
\hline \hline
\end{tabular}


TABLE IV. Projected relative errors for Higgs boson couplings and other Higgs observables with $2 \mathrm{ab}^{-1}$ of data at $250 \mathrm{GeV}$, comparing the cases of zero polarization, $80 \% e^{-}$ polarization and zero positron polarization, and $80 \% e^{-}$polarization and $30 \%$ positron polarization. In each case, the running is equally divided into two samples with opposite beam polarization orientation.

\begin{tabular}{lccc}
\hline \hline & No pol. & $80 \% / 0 \%$ & $80 \% / 30 \%$ \\
\hline$g(h b \bar{b})$ & 1.33 & 1.13 & 1.04 \\
$g(h c \bar{c})$ & 2.09 & 1.97 & 1.79 \\
$g(h g g)$ & 1.90 & 1.77 & 1.60 \\
$g(h W W)$ & 0.98 & 0.68 & 0.65 \\
$g(h \tau \tau)$ & 1.45 & 1.27 & 1.16 \\
$g(h Z Z)$ & 0.97 & 0.69 & 0.66 \\
$g(h \gamma \gamma)$ & 1.38 & 1.22 & 1.20 \\
$g(h \mu \mu)$ & 5.67 & 5.64 & 5.53 \\
$g(h b \bar{b}) / g(h W W)$ & 0.91 & 0.91 & 0.82 \\
$g(h W W) / g(h Z Z)$ & 0.07 & 0.07 & 0.07 \\
$\Gamma_{h}$ & 2.93 & 2.60 & 2.38 \\
$\sigma\left(e^{+} e^{-} \rightarrow Z h\right)$ & 0.78 & 0.78 & 0.70 \\
$\mathrm{BR}(h \rightarrow$ inv $)$ & 0.36 & 0.33 & 0.30 \\
$\mathrm{BR}(h \rightarrow$ other $)$ & 1.68 & 1.67 & 1.50 \\
\hline \hline
\end{tabular}

\section{INCLUSION OF $e^{+} e^{-}$DATA AT 500 GEV}

Our discussion so far has focused mainly on $e^{+} e^{-}$data that might be collected at $250 \mathrm{GeV}$. The ILC envisions a stage of running at $500 \mathrm{GeV}$. For CLIC, the current plan is to initially run at $380 \mathrm{GeV}$, with subsequent stages at $1 \mathrm{TeV}$ and above. Thus it is interesting to consider the effect of higher-energy data on this analysis.

There are three important effects of higher-energy running. First, the $W$ fusion process $e^{+} e^{-} \rightarrow \nu \bar{\nu} h$ turns on, providing a new source of data on Higgs cross sections and branching ratios. The dependence of this cross section on the parameter $\zeta_{W}$ is rather weak,

$$
\begin{aligned}
& \sigma\left(e^{+} e^{-} \rightarrow \nu \bar{\nu} h\right) /(S M) \\
& \quad= \begin{cases}\left(1+2 \eta_{W}-0.22 \zeta_{W}\right) & E_{C M}=250 \mathrm{GeV} \\
\left(1+2 \eta_{W}-0.34 \zeta_{W}\right) & E_{C M}=380 \mathrm{GeV} \\
\left(1+2 \eta_{W}-0.39 \zeta_{W}\right) & E_{C M}=500 \mathrm{GeV}\end{cases}
\end{aligned}
$$

Also, since the Higgs bosons from this reaction are not tagged, it is not possible to directly measure the absolute cross section. However, the $W$ fusion cross section is larger than the Higgsstrahlung cross section at $500 \mathrm{GeV}$, and the luminosity of linear colliders is expected to increase with energy, so this process adds a large amount of information on relative Higgs branching ratios. By combining the cross section measurements to specific final states with absolute branching ratio measurements at $250 \mathrm{GeV}$, one finds improved constraints on $\eta_{W}$ or $c_{H}$.

Second, as the center of mass energy increases, the angular distribution of $e^{+} e^{-} \rightarrow Z h$ predicted by the Standard Model evolves from one that is relatively flat to a $\sin ^{2} \theta$ distribution dominated by production of the longitudinal $Z$ polarization state. Since the contribution of the $\zeta_{Z}$ term is flat in angle and roughly independent of $Z$ polarization, this allows a much better discrimination of the $\eta_{Z}$ and $\zeta_{Z}$ contributions than at $250 \mathrm{GeV}$.

Third, the effects of dimension- 6 operators in $e^{+} e^{-} \rightarrow$ $W^{+} W^{-}$increases as $s / m_{W}^{2}$. Thus, running at higher energy allows stronger constraints on new physics effects in the triple gauge boson couplings, improving the error on the parameter $c_{W B}$ in the manner called for at the end of Sec. IV.

The results of a complete EFT fit including these effects is shown in the last column of Table III. This fit assumes $2 \mathrm{ab}^{-1}$ of data at $250 \mathrm{GeV}$ plus $4 \mathrm{ab}^{-1}$ at $500 \mathrm{GeV}$, divided equally between $e_{L}^{-} e_{R}^{+}$and $e_{R}^{-} e_{L}^{+}$polarized beams. The input measurements, with accuracies estimated by full simulation with the ILD detector model, are given in Appendix A.

\section{RECOGNITION OF NEW PHYSICS MODELS}

We can use the formalism presented in this paper to give quantitative estimates of the power of $e^{+} e^{-}$measurements of the Higgs boson to discover and discriminate models of new physics beyond the SM. From the large amount of literature on new physics modification of the Higgs couplings, we have chosen a selection of models that we feel are illustrative of possible new physics effects on the Higgs couplings. In this section, we will compare their predictions for Higgs couplings to the ILC error estimates computed in this paper.

The EFT fit presented here can be used to estimate the significance of the observation of Higgs coupling deviations from the SM and discrimination of the effects of different models. Our method makes use of the linear dependence of the Higgs couplings on the EFT coefficients. Consider as observables $\mathcal{O}_{i}$ the Higgs couplings obtained from the fits described in this paper. Each coupling has an expansion in EFT coefficients of the form (17) with coefficients $V_{i J}$. Let $\delta g_{i}$ be the Higgs couplings deviations predicted in a given model, arranged as a vector. Let $C_{J K}$ be the covariance matrix of the variables $c_{J}$ determined by the fit. Then

$$
\left(\chi^{2}\right)=g^{T}\left[V C V^{T}\right]^{-1} g
$$

gives the $\chi^{2}=2 \log$ likelihood testing the goodness of fit for this model relative to the Standard Model. Similarly, if $g_{A}$ and $g_{B}$ are two such vectors for models $A$ and $B$, 


$$
\left(\chi^{2}\right)_{A B}=\left(g_{A}^{T}-g_{B}^{T}\right)\left[V C V^{T}\right]^{-1}\left(g_{A}-g_{B}\right)
$$

gives the $\chi^{2}$ for $A$ given the hypothesis $B$ or vice versa. The significance in $\sigma$ of the deviation of a model from the SM, or of one model from another, is roughly the square root of the $\chi^{2}$ computed in this way.

It will always be true that some models of new physics are observable through Higgs coupling deviations while others are not. All viable models of new physics beyond the SM exhibit "decoupling." That is, as the new particle masses are increased, the predicted deviations from the SM in the Higgs couplings and in other precision observables tend to zero. It is interesting to ask, though, whether there are models that predict significant deviations in the Higgs couplings for parameter values at which the new particles are very heavy, outside the reach of the LHC. A systematic study of supersymmetric models [37,38] shows that there are a significant number of such models. In fact, Figs. 1-5 of [37] show that constraints on the Higgs couplings probe the model space in a direction roughly orthogonal to that probed by direct particle searches. It makes sense, then, to open this new line of attack on the problem of discovering physics beyond the SM.

To illustrate the range of new physics models that can be found through studies of the Higgs couplings, we present a list of nine models with significant Higgs boson coupling deviations in which the new particles present in the model are unlikely to be discoverable at the LHC, even in the high-luminosity era. These models are:

(1) A supersymmetric model, model \#1259073 of the Phenomenological Minimal Supersymmetric Standard Model (PMSSM) models described in [37]. This model has relatively high colored supersymmetry (SUSY) particle masses: $m(\tilde{b})=3.4 \mathrm{TeV}$, $m(\tilde{g})=4 \mathrm{TeV}$, but still these shift the $h b \bar{b}$ coupling significantly. The lightest SUSY particles are a Higgsino multiplet at $515 \mathrm{GeV}$.

(2) A type II 2-Higgs-doublet model from [39], with heavy Higgs bosons at $600 \mathrm{GeV}$ and $\tan \beta=7$.
Higgs couplings are evaluated with one-loop corrections as in [40]. This model and the next two lie in the wedge-shaped region where the LHC has limited sensitivity.

(3) A type X 2-Higgs-doublet model from [39], with heavy Higgs bosons at $450 \mathrm{GeV}$ and $\tan \beta=6$. Higgs couplings are evaluated with one-loop corrections as in [40].

(4) A type Y 2-Higgs-doublet model from [39], with heavy Higgs bosons at $600 \mathrm{GeV}$ and $\tan \beta=7$. Higgs couplings are evaluated with one-loop corrections as in [40].

(5) A composite Higgs model MCHM5 with $f=1.2 \mathrm{TeV}$, described in [41]. The lightest new particle in this model is a vectorlike top quark partner $T$ at $1.7 \mathrm{TeV}$. However, the single production cross section for this particle can be very small.

(6) A Little Higgs model with T-parity in the family of models considered in [42], with $f=785 \mathrm{GeV}$ and the top quark partner $T$ at $2 \mathrm{TeV}$. (This model is on the boundary with respect to precision electroweak.)

(7) A Little Higgs model with T-parity described in [43], with $f=1 \mathrm{TeV}$ and the option $\mathrm{B}$ for light-quark Yukawa couplings. In this model, the top quark partner $T$ has a mass of $2.03 \mathrm{TeV}$.

(8) A Higgs-radion mixing model described in [44]. The radion mass is taken to be $500 \mathrm{GeV}$; other relevant extra-dimensional states can be at multi-TeV masses.

(9) A model with a Higgs singlet added to the Standard Model to allow electroweak baryogenesis and to provide a portal to the dark matter sector, described in [45]. The singlet mass is $2.8 \mathrm{TeV}$, with mixing as large as permitted by decoupling.

The coupling deviations in these models are listed in Table V. These deviations are shown graphically in Appendix B, together with the uncertainties that would result from the fit to ILC data at 250 and $500 \mathrm{GeV}$.

Comparing these models to the Standard Model, we find the following $\chi^{2}$ separation. Using the fit of Sec. IV, for the

TABLE V. Deviations from the Standard Model predictions for the Higgs boson couplings, in percent, for the set of new physics models described in the text. As in Table I, the effective couplings $g(h W W)$ and $g(h Z Z)$ are defined as proportional to the square roots of the corresponding partial widths.

\begin{tabular}{llcccccccc}
\hline \hline \multicolumn{1}{c}{ Model } & \multicolumn{1}{c}{$b \bar{b}$} & $c \bar{c}$ & $g g$ & $W W$ & \multicolumn{1}{c}{$\tau \tau$} & \multicolumn{1}{c}{ ZZ } & \multicolumn{1}{c}{$\gamma \gamma$} & $\mu \mu$ \\
\hline 1 & MSSM [37] & +4.8 & -0.8 & -0.8 & -0.2 & +0.4 & -0.5 & +0.1 & +0.3 \\
2 & Type II 2HD [39] & +10.1 & -0.2 & -0.2 & 0.0 & +9.8 & 0.0 & +0.1 & +9.8 \\
3 & Type X 2HD [39] & -0.2 & -0.2 & -0.2 & 0.0 & +7.8 & 0.0 & 0.0 & +7.8 \\
4 & Type Y 2HD [39] & +10.1 & -0.2 & -0.2 & 0.0 & -0.2 & 0.0 & 0.1 & -0.2 \\
5 & Composite Higgs [41] & -6.4 & -6.4 & -6.4 & -2.1 & -6.4 & -2.1 & -2.1 & -6.4 \\
6 & Little Higgs with T-parity [42] & 0.0 & 0.0 & -6.1 & -2.5 & 0.0 & -2.5 & -1.5 & 0.0 \\
7 & Little Higgs with T-parity [43] & -7.8 & -4.6 & -3.5 & -1.5 & -7.8 & -1.5 & -1.0 & -7.8 \\
8 & Higgs radion [44] & -1.5 & -1.5 & +10.0 & -1.5 & -1.5 & -1.5 & -1.0 & -1.5 \\
9 & Higgs singlet [45] & -3.5 & -3.5 & -3.5 & -3.5 & -3.5 & -3.5 & -3.5 & -3.5 \\
\hline \hline
\end{tabular}




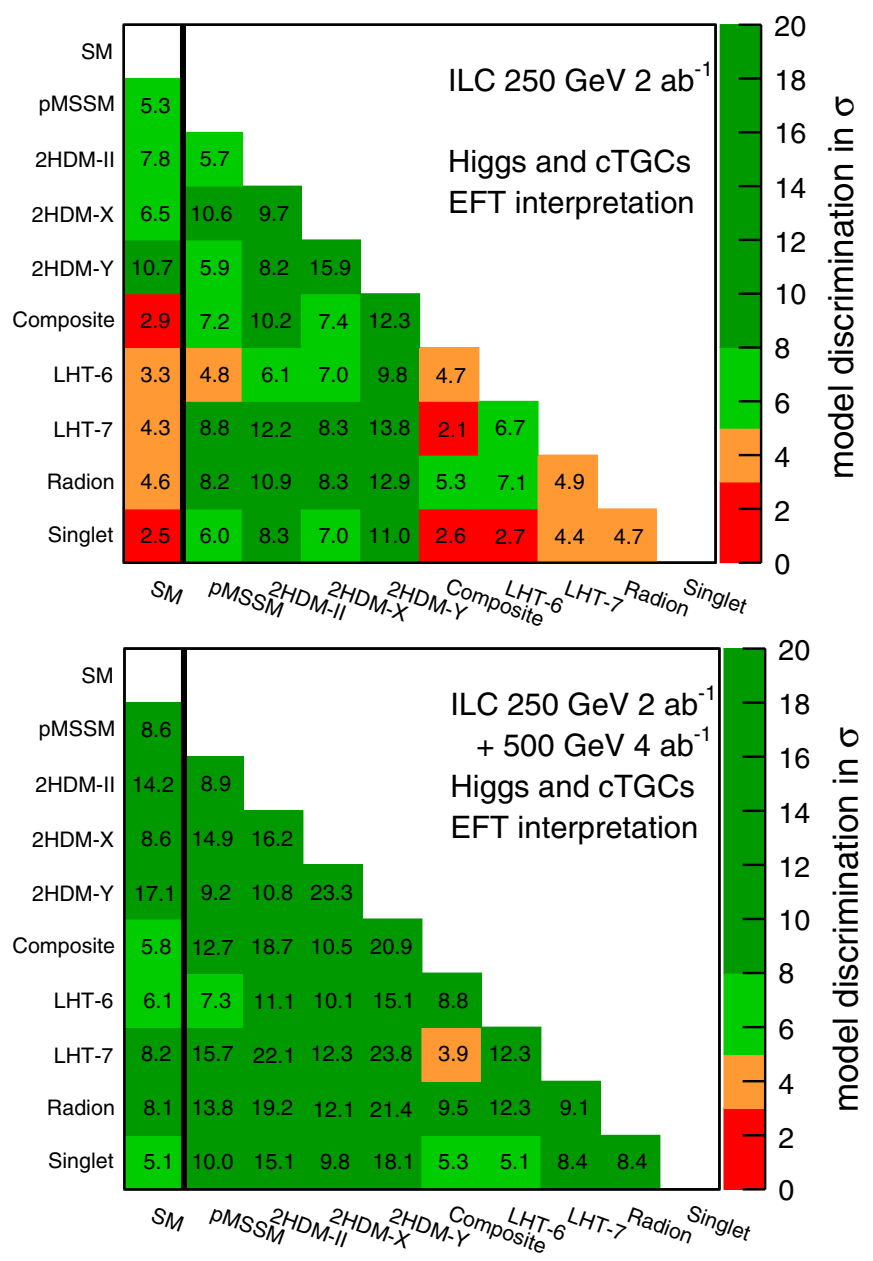

FIG. 2. Graphical representation of the $\chi^{2}$ separation of the Standard Model and the models 1-9 described in the text: (a) with $2 \mathrm{ab}^{-1}$ of data at the ILC at $250 \mathrm{GeV}$; (b) with $2 \mathrm{ab}^{-1}$ of data at the ILC at $250 \mathrm{GeV}$ plus $4 \mathrm{ab}^{-1}$ of data at the ILC at $500 \mathrm{GeV}$. Comparisons in orange have above $3 \sigma$ separation; comparison in green have above $5 \sigma$ separation; comparisons in dark green have above $8 \sigma$ separation.

ILC at $250 \mathrm{GeV}$ with $2 \mathrm{ab}^{-1}$ of data, the relative $\chi^{2} \mathrm{~s}$ of the models are:

\begin{tabular}{lrrrrrrrrrr}
\hline & SM & 1 & 2 & 3 & 4 & 5 & 6 & 7 & 8 & 9 \\
\hline SM & 0 & 29 & 63 & 43 & 115 & 10 & 12 & 20 & 22 & 7.3 \\
1 & 29 & 0 & 34 & 113 & 36 & 53 & 24 & 78 & 68 & 37 \\
2 & 63 & 34 & 0 & 95 & 68 & 105 & 39 & 149 & 120 & 71 \\
3 & 43 & 113 & 95 & 0 & 256 & 57 & 51 & 71 & 70 & 50 \\
4 & 115 & 36 & 68 & 256 & 0 & 152 & 97 & 191 & 167 & 123 \\
5 & 10 & 53 & 105 & 57 & 152 & 0 & 23 & 5.5 & 29 & 8.3 \\
6 & 12 & 24 & 39 & 51 & 97 & 23 & 0 & 46 & 51 & 8.8 \\
7 & 20 & 78 & 149 & 71 & 191 & 5.5 & 46 & 0 & 26 & 21 \\
8 & 22 & 68 & 120 & 70 & 167 & 29 & 51 & 26 & 0 & 23 \\
9 & 7.3 & 37 & 71 & 50 & 123 & 8.3 & 8.8 & 21 & 23 & 0 \\
\hline
\end{tabular}

Every model except \#9 is separated from the Standard Model by at least $3 \sigma$, and the models are generally separated from one another by a comparable amount [46]. The $\sigma$ separations of the models from the SM and from one another are illustrated in Fig. 2(a).

Using the fit of Sec. VI, for the ILC at $250 \mathrm{GeV}$ with $2 \mathrm{ab}^{-1}$ of data and then at $500 \mathrm{GeV}$ with $4 \mathrm{ab}^{-1}$ of data, the relative $\chi^{2}$ s of the models are:

\begin{tabular}{lrrrrrrrrrr}
\hline & SM & 1 & \multicolumn{1}{c}{2} & \multicolumn{1}{c}{3} & \multicolumn{1}{c}{4} & 5 & 6 & 7 & 8 & 9 \\
\hline SM & 0 & 75 & 204 & 75 & 295 & 35 & 39 & 68 & 67 & 27 \\
1 & 75 & 0 & 80 & 222 & 85 & 162 & 55 & 247 & 193 & 101 \\
2 & 204 & 80 & 0 & 263 & 118 & 352 & 124 & 492 & 371 & 230 \\
3 & 75 & 222 & 263 & 0 & 543 & 113 & 104 & 152 & 147 & 97 \\
4 & 295 & 85 & 118 & 543 & 0 & 438 & 230 & 568 & 458 & 329 \\
5 & 35 & 162 & 352 & 113 & 438 & 0 & 78 & 17 & 93 & 30 \\
6 & 39 & 55 & 124 & 104 & 230 & 78 & 0 & 153 & 154 & 27 \\
7 & 68 & 247 & 492 & 152 & 568 & 17 & 153 & 0 & 85 & 72 \\
8 & 67 & 193 & 371 & 147 & 458 & 93 & 154 & 85 & 0 & 71 \\
9 & 27 & 101 & 230 & 97 & 329 & 30 & 27 & 72 & 71 & 0 \\
\hline
\end{tabular}

With this data set, the various models are distinguished from the Standard Model at least $5 \sigma$. Except in two cases, the models are also well distinguished from one another, so that the results give a clear indication of the nature of the new physics that has been discovered through the Higgs coupling deviations. The $\sigma$ separations of the models from the SM and from one another are illustrated in Fig. 2(b).

These examples illustrate the ability of future $e^{+} e^{-}$ colliders to expose models of new physics even in cases in which the new particles are beyond the reach of direct searches at the LHC.

\section{CONCLUSIONS}

In this paper, we have presented an improved method for the extraction of model-independent Higgs couplings from the data that will be provided by future $e^{+} e^{-}$colliders. We began by explaining that a simple parametrization of new physics effects by rescaling the Standard Model Higgs couplings by $\kappa_{I}$ parameters is not sufficiently general to encompass the full range of models of new physics. Instead, we advocate the description of new physics effects on Higgs couplings by the coefficients of dimension- 6 operators that can be added to the Lagrangian of the Standard Model. This effective field theory description encompasses a broader range of new physics effects. At the same time, it brings in new constraints from $S U(2) \times U(1)$ gauge invariance and from Higgs cross section measurements not previously considered in fits for Higgs coupling. This method draws information from precision electroweak measurements, $e^{+} e^{-} \rightarrow W^{+} W^{-}$, and precision Higgs measurements, thus taking full advantage of the richness of the information provided by future $e^{+} e^{-}$colliders.

Using this approach, we have analyzed the expectations of the ILC and other proposed colliders for extracting the couplings of the Higgs boson with percent-level accuracy and for observing and discriminating models of new physics whose new particles are beyond the reach of 
LHC. This program gives a powerful avenue to the discovery of physics beyond the Standard Model.

\section{ACKNOWLEDGMENTS}

We benefited very much from discussion of this work with many colleagues, including Halina Abramowicz, Jim Brau, Nathaniel Craig, Erez Etzion, Howard Haber, Sho Iwamoto, Gabriel Lee, Maxim Perelstein, Philipp Roloff, Yael Shadmi, and Tomohiko Tanabe. We are grateful to Christophe Grojean, Ahmed Ismail, Mihoko Nojiri, Maxim Perelstein, and Shinya Kanemura for detailed discussions of the models analyzed in Sec. VII. We are especially grateful to Gauthier Durieux, Christophe Grojean, Jiayin Gu, and Kechen Wang for sharing with us insights from [13] that were crucial to this work, and for much other useful advice about the application of the EFT formalism. M. E. P. thanks Kirsten Sachs for arranging a visit to DESY that was essential to this collaboration. T. B., S. J., and M. E. P. were supported by the U.S. Department of Energy under Contract No. DE-AC02-76SF00515. T. B. was also supported by a KEK Short-Term Invited Fellowship. He thanks the KEK ILC group for hospitality during this visit. S. J. was also supported by the National Research Foundation of Korea under Grants No. 2015R1A4A1042542 and No. 2017R1D1A1B03030820. K. F. and T. O. were supported by the Japan Society for the Promotion of Science (JSPS) under Grants-in-Aid for Science Research No. 16H02173 and No. 16H02176. J. T. was supported by JSPS under Grant-in-Aid No. 15H02083. R. K. and J. L. were supported by the Deutsche Forschungsgemeinschaft (DFG) through the Sonderforschungsbereich (SFB) 676 Particles, Strings and the Early Universe, project B1.

\section{APPENDIX A: ERROR ESTIMATES INPUT INTO THE FITS PRESENTED IN THIS PAPER}

The fits presented in this paper rely on error estimates for precision electroweak, $W$ boson, and Higgs boson measurements. In particular, they rely heavily on uncertainties estimated for measurements that can be carried out at the ILC at 250, 350 and $500 \mathrm{GeV}$. In this section, we provide tables of the uncertainties we have assumed in the fits present here. These uncertainties are based on fullsimulation studies done for the SiD and ILD detector models used for linear collider experimental studies at the ILC and CEPC. We also specify the additional inputs from precision electroweak and LHC measurements of Higgs branching ratios that are used in our analysis.

Table VI gives the expected statistical errors on Higgs cross section and branching ratio measurements for polarized beams and for luminosity samples of $250 \mathrm{fb}^{-1}$. The numbers given in Table VI are statistical errors only. They can be rescaled to any luminosity by dividing by the square root of the integrated luminosity. In our fits, we have added the statistical error of each measurement for Higgs observables $(\sigma$ or $\sigma \cdot B R$ ) in quadrature with two types of assumed common
TABLE VI. Projected statistical errors, in percent, for Higgs boson measurements input to our fits. The errors are quoted for luminosity samples of $250 \mathrm{fb}^{-1}$ for $e^{+} e^{-}$beams with $-80 \%$ electron polarization and $+30 \%$ positron polarization, in the top half of the table, and with $+80 \%$ electron polarization and $-30 \%$ positron polarization, in the bottom half of the table. Except for the first and last segments of each set, these are measurements of $\sigma \cdot B R$, relative to the Standard Model expectation. The top lines give the error for the total cross section relative to the Standard Model and the $95 \%$ confidence upper limit on the branching ratio for Higgs to invisible decays. The bottom lines in each half give the expected errors on the $a$ and $b$ parameters and their correlation (all in percent) for $e^{+} e^{-} \rightarrow Z h$ (see (11). All error estimates in this table are based on full simulation, with the exception of entries marked with a ${ }^{*}$, which are based on extrapolation from full simulation results.

\begin{tabular}{|c|c|c|c|c|c|c|}
\hline \multicolumn{7}{|c|}{$-80 \% e^{-},+30 \% e^{+}$polarization: } \\
\hline & \multicolumn{2}{|l|}{$250 \mathrm{GeV}$} & $350 \mathrm{GeV}$ & \multicolumn{3}{|c|}{$500 \mathrm{GeV}$} \\
\hline & $Z h$ & $\nu \bar{\nu} h$ & $Z h$ & $\nu \bar{\nu} h$ & $Z h$ & $\nu \bar{\nu} h$ \\
\hline$\sigma[47-50]$ & 2.0 & & 1.8 & & 4.2 & \\
\hline$h \rightarrow$ invisible $[51,52]$ & 0.86 & & 1.4 & & 3.4 & \\
\hline$h \rightarrow b \bar{b}[53-56]$ & 1.3 & 8.1 & 1.5 & 1.8 & 2.5 & 0.93 \\
\hline$h \rightarrow c \bar{c}[53,54]$ & 8.3 & & 11 & 19 & 18 & 8.8 \\
\hline$h \rightarrow g g[53,54]$ & 7.0 & & 8.4 & 7.7 & 15 & 5.8 \\
\hline$h \rightarrow W W[56-58]$ & 4.6 & & $5.6 *$ & $5.7 *$ & 7.7 & 3.4 \\
\hline$h \rightarrow \tau \tau$ [59] & 3.2 & & $4.0 *$ & $16 *$ & 6.1 & 9.8 \\
\hline$h \rightarrow Z Z$ [2] & 18 & & $25 *$ & $20 *$ & $35 *$ & $12 *$ \\
\hline$h \rightarrow \gamma \gamma[60]$ & $34 *$ & & $39 *$ & $45 *$ & 47 & 27 \\
\hline$h \rightarrow \mu \mu[61,62]$ & $72 *$ & & $87 *$ & $160 *$ & $120 *$ & $100 *$ \\
\hline$a[24]$ & 7.6 & & $2.7 *$ & & 4.0 & \\
\hline$b$ & 2.7 & & $0.69 *$ & & 0.70 & \\
\hline$\rho(a, b)$ & -99.17 & & $-95.6 *$ & & -84.8 & \\
\hline \multicolumn{7}{|c|}{$+80 \% e^{-},-30 \% e^{+}$polarization: } \\
\hline & $250 \mathrm{GeV}$ & & $350 \mathrm{GeV}$ & & $500 \mathrm{GeV}$ & \\
\hline & $Z h$ & $\nu \bar{\nu} h$ & $Z h$ & $\nu \bar{\nu} h$ & $Z h$ & $\nu \bar{\nu} h$ \\
\hline$\sigma$ & 2.0 & & 1.8 & & 4.2 & \\
\hline$h \rightarrow$ invisible & 0.61 & & 1.3 & & 2.4 & \\
\hline$h \rightarrow b \bar{b}$ & 1.3 & 33 & 1.5 & 7.5 & 2.5 & 3.8 \\
\hline$h \rightarrow c \bar{c}$ & 8.3 & & 11 & 79 & 18 & 36 \\
\hline$h \rightarrow g g$ & 7.0 & & 8.4 & 32 & 15 & 24 \\
\hline$h \rightarrow W W$ & 4.6 & & 5.6 & 24 & 7.7 & 14 \\
\hline$h \rightarrow \tau \tau$ & 3.2 & & 4.0 & 66 & 6.1 & 40 \\
\hline$h \rightarrow Z Z$ & 18 & & 25 & 81 & 35 & 48 \\
\hline$h \rightarrow \gamma \gamma$ & 34 & & 39 & 180 & 47 & 110 \\
\hline$h \rightarrow \mu \mu$ & 72 & & 87 & 670 & 120 & 420 \\
\hline$a$ & 9.1 & & $3.1 *$ & & 4.2 & \\
\hline$b$ & 3.2 & & $0.79 *$ & & 0.75 & \\
\hline$\rho(a, b)$ & -99.39 & & $-96.6 *$ & & -86.5 & \\
\hline
\end{tabular}

systematic errors, $1.0 \times 10^{-3}$ from theory prediction, and $1.0 \times 10^{-3}$ from luminosity and beam polarizations measurements [30]. For the $h \rightarrow b \bar{b}$ observables, we have also added in quadrature an additional systematic error from $b$-tagging efficiency, taken to be $3.0 \times 10^{-3} \times \sqrt{250 / L}$ ( $L$ for integrated luminosities in $\mathrm{fb}^{-1}$ ) [2]. 
The first two lines of Table 6 give estimates of the expected error in the total $e^{+} e^{-} \rightarrow Z h$ cross section and the expected 95\% confidence level upper limit on Higgs to invisible decays, assuming the Standard Model predictions. The following entries give the estimated errors on $\sigma \times B R$ measurements to the given final states, using the reactions $e^{+} e^{-} \rightarrow Z h$ and $e^{+} e^{-} \rightarrow \nu \bar{\nu} h$. The uncertainties quoted here are for polarized $e^{+} e^{-}$beams with $-80 \%$ electron and $+30 \%$ positron polarization. References to the original studies are given in each line. Most of the estimates in this table are identical to the ones reported in the article "ILC Operating Scenarios" [33], a few of them have been updated since then by new full simulation studies and are briefly described in the following. The estimates for $\sigma_{Z h}$. $B R_{W W}$ are improved by a factor of 1.4 at $\sqrt{s}=250 \mathrm{GeV}$, after adding the contributions from $Z \rightarrow l l, W W^{*} \rightarrow$ $l \nu l \nu / l \nu 2 q / 4 q$ channels [58], and by a factor of 1.7 at $\sqrt{s}=500 \mathrm{GeV}$, after adding the contributions from $Z \rightarrow q q, W W^{*} \rightarrow 4 q$ channels [63]. The estimates for $\sigma_{Z h}$. $B R_{b b}$ and $\sigma_{\nu \nu h} \cdot B R_{b b}$ at $\sqrt{s}=250 \mathrm{GeV}$ are updated based on new analysis performed using ILD Detailed Baseline Design (DBD) simulation and reconstruction tools [55], and, more importantly, the correlation between them, which is $-34 \%$, is now incorporated into the fit. The estimates for $\sigma_{\nu \nu h} \cdot B R_{b b / c c / g g}$ at $\sqrt{s}=350 \mathrm{GeV}$ are updated based on new full simulation results in [54]. The estimates for $\sigma_{Z h / \nu \nu h} \cdot B R_{\tau \tau}$ are updated to the published results [59]. The up-to-date references for all of the estimates are indicated in the table.

For the $e^{+} e^{-}$beams with $+80 \%$ electron and $-30 \%$ positron polarizations, we generally assume that the expected errors are independent of the polarization state, with a slightly higher cross section for $e_{L}^{-} e_{R}^{+}$being compensated by slightly lower backgrounds for $e_{R}^{-} e_{L}^{+}$. Dedicated studies for $+80 \%$ electron and $-30 \%$ positron polarization were done for the $h \rightarrow$ invisible and $a, b$ measurements, and here we quote the analysis results directly. The $W W$ fusion process $e^{+} e^{-} \rightarrow \nu \bar{\nu} h$ requires the $e_{L}^{-} e_{R}^{+}$initial state, and so the rate is much smaller in this polarization configuration. The errors quoted in the table are obtained by multiplying the corresponding errors in the top half of the table by 4.1, the inverse of the square root of the relative $e_{L}^{-} e_{R}^{+}$luminosity.

The errors on the total cross section and angular distribution in $e^{+} e^{-} \rightarrow Z h$ are usefully quoted as errors on the $a$ and $b$ parameters defined in (11) and in [15]. The expected errors on $a$ and $b$ and their correlation $\rho(a, b)$ are shown in Table VI. For $\sqrt{s}=250$ and $500 \mathrm{GeV}$, these errors have been estimated in [24] from ILD full-simulation studies for both polarization configurations. For $\sqrt{s}=350 \mathrm{GeV}$, we have estimated these errors by interpolation.

The expected errors on the anomalous triple gauge coupling (TGC) parameters of $e^{+} e^{-} \rightarrow W^{+} W^{-}$are shown in Table VII. These errors are based on extrapolation from the studies of $[29,30]$, taking into account the dependences
TABLE VII. Projected statistical errors, in percent, for $e^{+} e^{-} \rightarrow$ $W^{+} W^{-}$measurements input to our fits. The errors are quoted for luminosity samples of $500 \mathrm{fb}^{-1}$ divided equally between beams with $-80 \%$ electron polarization and $+30 \%$ positron polarization and brams with $+80 \%$ electron polarization and $-30 \%$ positron polarization. The last three lines give the correlation coefficients, also in percent. All error estimates in this table are based on full simulation using the ILD or $\mathrm{SiD}$ detector model, with the exception of entries marked with a ${ }^{*}$, which are based on extrapolation from full simulation results.

\begin{tabular}{lccc}
\hline \hline & $\begin{array}{c}250 \mathrm{GeV} \\
W^{+} W^{-}\end{array}$ & $\begin{array}{c}350 \mathrm{GeV} \\
W^{+} W^{-}\end{array}$ & $\begin{array}{c}500 \mathrm{GeV} \\
W^{+} W^{-}\end{array}$ \\
\hline$g_{1 Z}$ & $0.062 *$ & $0.033 *$ & 0.025 \\
$\kappa_{A}$ & $0.096 *$ & $0.049 *$ & 0.034 \\
$\lambda_{A}$ & $0.077 *$ & $0.047 *$ & 0.037 \\
$\rho\left(g_{1 Z}, \kappa_{A}\right)$ & $63.4 *$ & $63.4 *$ & 63.4 \\
$\rho\left(g_{1 Z}, \lambda_{A}\right)$ & $47.7 *$ & $47.7 *$ & 47.7 \\
$\rho\left(\kappa_{A}, \lambda_{A}\right)$ & $35.4 *$ & $35.4 *$ & 35.4 \\
\hline \hline
\end{tabular}

on $\sqrt{s}$, statistics and detector acceptances [64]. Furthermore, since the studies in [29,30] are performed using a binned fit for three angular distributions in the semileptonic channel, an additional scaling factor, 1.6-2, is applied to the extrapolations, to take into account the potential improvement from an unbinned fit for five angular distributions in both semileptonic and full hadronic channels. The errors for TGCs are quoted for samples of $500 \mathrm{fb}^{-1}$ of data, divided equally between the two polarization states. For simplicity, we use the same estimates for the errors at unpolarized colliders. In our analysis, we have added to these statistical errors the systematic errors $0.3 \times 10^{-3}$ for both $g_{1 Z}$ and $\kappa_{A}$, and $0.2 \times 10^{-3}$ for $\lambda_{A}$ [64].

TABLE VIII. Values and uncertainties for precision electroweak observables used in this paper. The values are taken from [65], except for the averaged value of $A_{\ell}$, which corresponds to the averaged value of $\sin ^{2} \theta_{\text {eff }}$ in [67]. The best fit values are those of the fit in [65]. For the purpose of fitting Higgs boson couplings as described in Sec. VII, we use improvements in some of the errors expected from LHC [66] and ILC [3]. The improved estimate of the $W$ width is obtained from $\Gamma_{W}=$ $\Gamma(W \rightarrow \ell \nu) / \mathrm{BR}(W \rightarrow \ell \nu)$.

\begin{tabular}{lcccc}
\hline \hline Observable & $\begin{array}{c}\text { Current } \\
\text { value }\end{array}$ & Current $\sigma$ & Future $\sigma$ & $\begin{array}{c}\text { SM best } \\
\text { fit value }\end{array}$ \\
\hline$\alpha^{-1}\left(m_{Z}^{2}\right)$ & 128.9220 & 0.0178 & & (same) \\
$G_{F}\left(10^{-10} \mathrm{GeV}^{-2}\right)$ & 1166378.7 & 0.6 & & (same) \\
$m_{W}(\mathrm{MeV})$ & 80385 & 15 & 5 & 80361 \\
$m_{Z}(\mathrm{MeV})$ & 91187.6 & 2.1 & & 91.1880 \\
$m_{h}(\mathrm{MeV})$ & 125090 & 240 & 15 & 125110 \\
$A_{\ell}$ & 0.14696 & 0.0013 & & 0.147937 \\
$\Gamma_{\ell}(\mathrm{MeV})$ & 83.984 & 0.086 & & 83.995 \\
$\Gamma_{Z}(\mathrm{MeV})$ & 2495.2 & 2.3 & & 2.4943 \\
$\Gamma_{W}(\mathrm{MeV})$ & 2085 & 42 & 2 & 2.0888 \\
\hline \hline
\end{tabular}


The precision electroweak inputs to our fit are shown in Table VIII. For most of the entries, we have assumed the current uncertainties, from the Particle Data Group compilation [65]. For three of the values, we have assumed improvements in uncertainties: for the $W$ mass, from LHC [66], for the Higgs boson mass, from ILC [47], and, for the $W$ width, from $\Gamma_{W}=\Gamma(W \rightarrow \ell \nu) / \mathrm{BR}(W \rightarrow \ell \nu)$, using the theoretical value of $\Gamma(W \rightarrow \ell \nu)$ from our fit and the value of $\mathrm{BR}(W \rightarrow \ell \nu)$ that will be measured at the ILC at $250 \mathrm{GeV}$ with $10^{7} \mathrm{~W}$ pair events.

We have input the following errors on ratios of branching ratios from the LHC, as described in Sec. III:
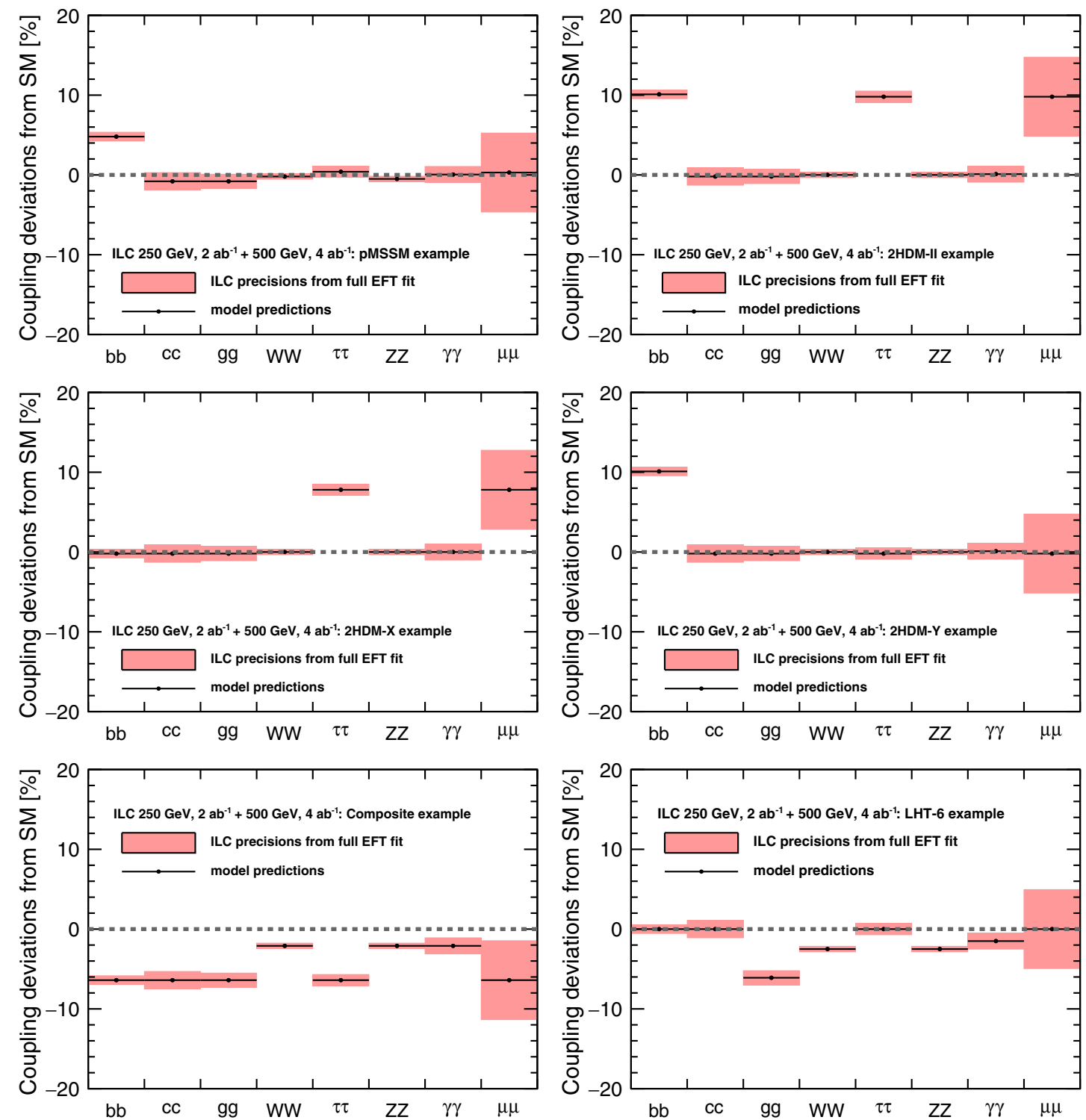

FIG. 3. Visualization of the deviations of Higgs couplings from the SM for the new physics models 1-6 discussed in Sec. VII, compared to the uncertainties in the measurements expected from a fit to ILC data at 250 and $500 \mathrm{GeV}$. 


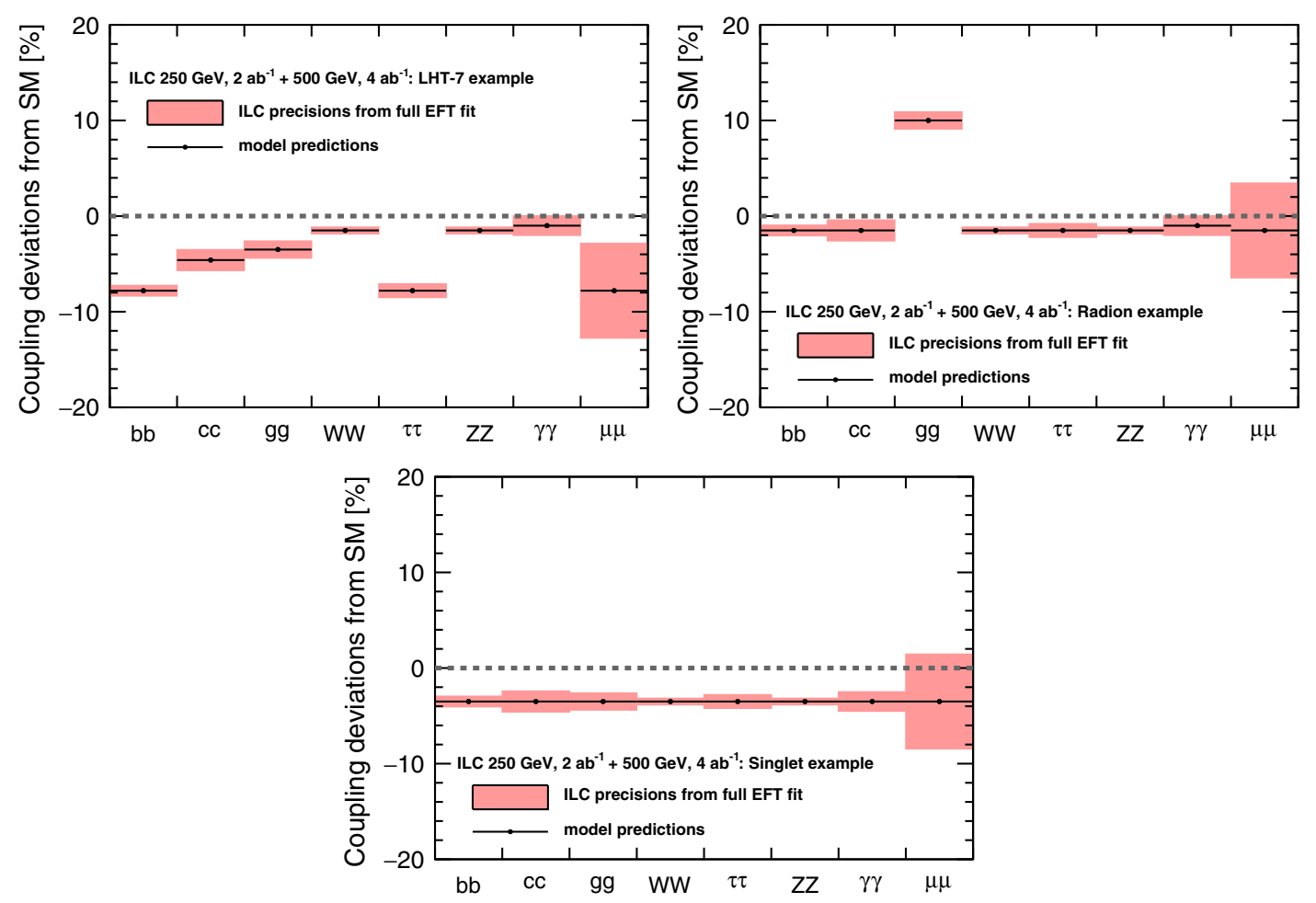

FIG. 4. Visualization of the deviations of Higgs couplings from the SM for the new physics models 7-9 discussed in Sec. VII, compared to the uncertainties in the measurements expected from a fit to ILC data at 250 and $500 \mathrm{GeV}$.

improve the measurement of $\sigma_{Z h} \cdot B R_{W W}$ at $250 \mathrm{GeV}$ by including contribution from full hadronic channels. Second, we plan to improve the constraints on $h \gamma Z$ couplings by adding measurements of the branching ratio for $h \rightarrow \gamma Z$ ) and the cross section for $e^{+} e^{-} \rightarrow \gamma h$. Third, we plan to improve the constraints on the EFT coefficients $c_{H L}, c_{H L}^{\prime}, c_{H E}$, by measuring the cross sections of $e^{+} e^{-} \rightarrow$ $\gamma Z$ for both left- and right-handed beams. Finally, we plan to carry out a full analysis of the measurement of the branching ratio for $W \rightarrow e \nu$ which should improve on the estimate given in Table VIII.

\section{APPENDIX B: ILLUSTRATION OF THE DISCRIMINATION OF MODELS BY PRECISION HIGGS MEASUREMENTS}

Figures 3 and 4 show the deviations from the Standard Model expectations for the Higgs boson couplings, in percent, expected for each of the models considered in Sec. VII, along with the uncertainties that would result from the fit to ILC data at 250 and $500 \mathrm{GeV}$ described in Sec. VI. Note that these uncertainties are correlated, and that these correlations are taken into account in the $\sigma$ values that we quote in Sec. VII.
[1] H. Baer et al., The International Linear Collider technical design report—Volume 2: Physics, arXiv:1306.6352.

[2] D. M. Asner et al., ILC Higgs White Paper, arXiv:1310.0763.

[3] K. Fujii et al., Physics case for the International Linear Collider, arXiv:1506.05992.

[4] M. Bicer et al. (TLEP Design Study Working Group), First look at the physics case of TLEP, J. High Energy Phys. 01 (2014) 164.

[5] M. Ahmad et al. (CEPC-SPPC Study Group), Reports No. IHEP-CEPC-DR-2015-01, No. IHEP-TH-2015-01, and No. IHEP-EP-2015-01.
[6] H. Abramowicz et al., Higgs physics at the CLIC electron-positron linear collider, Eur. Phys. J. C 77, 475 (2017).

[7] R. Lafaye, T. Plehn, M. Rauch, D. Zerwas, and M. Duhrssen, Measuring the Higgs sector, J. High Energy Phys. 08 (2009) 009; R. Lafaye, T. Plehn, M. Rauch, and D. Zerwas, Higgs factories: Higgs-Strahlung versus W-fusion, Phys. Rev. D 96, 075044 (2017).

[8] G. F. Giudice, C. Grojean, A. Pomarol, and R. Rattazzi, The strongly interacting light Higgs, J. High Energy Phys. 06 (2007) 045. 
[9] R. Contino, M. Ghezzi, C. Grojean, M. Muhlleitner, and M. Spira, Effective Lagrangian for a light Higgs-like scalar, J. High Energy Phys. 07 (2013) 035.

[10] N. Craig, J. Gu, Z. Liu, and K. Wang, Beyond higgs couplings: Probing the higgs with angular observables at future $e^{+} e^{-}$Colliders, J. High Energy Phys. 03 (2016) 050.

[11] S. F. Ge, H. J. He, and R. Q. Xiao, Probing new physics scales from higgs and electroweak observables at $e^{+} e^{-}$ higgs factory, J. High Energy Phys. 10 (2016) 007.

[12] J. Ellis, P. Roloff, V. Sanz, and T. You, Dimension-6 operator analysis of the CLIC sensitivity to new physics, J. High Energy Phys. 05 (2017) 096.

[13] G. Durieux, C. Grojean, J. Gu, and K. Wang, The leptonic future of the Higgs, J. High Energy Phys. 09 (2017) 014.

[14] D. de Florian et al. (LHC Higgs Cross Section Working Group), Handbook of LHC Higgs cross sections: 4. Deciphering the nature of the Higgs sector, arXiv:1610.07922.

[15] T. Barklow, K. Fujii, S. Jung, M. E. Peskin, and J. Tian, following article, Model-independent determination of the triple Higgs coupling at $e^{+} e^{-}$colliders, Phys. Rev. D 97, 053004 (2018).

[16] B. Grzadkowski, M. Iskrzynski, M. Misiak, and J. Rosiek, Dimension-six terms in the Standard Model Lagrangian, J. High Energy Phys. 10 (2010) 085.

[17] N. Craig, M. Farina, M. McCullough, and M. Perelstein, Precision Higgsstrahlung as a probe of new physics, J. High Energy Phys. 03 (2015) 146.

[18] J. Elias-Mir, J. R. Espinosa, E. Masso, and A. Pomarol, Renormalization of dimension-six operators relevant for the Higgs decays $h \rightarrow \gamma \gamma, \gamma Z$, J. High Energy Phys. 08 (2013) 033.

[19] M.E. Peskin and T. Takeuchi, A New Constraint on a Strongly Interacting Higgs Sector, Phys. Rev. Lett. 65, 964 (1990); Estimation of oblique electroweak corrections, Phys. Rev. D 46, 381 (1992).

[20] V. Barger, T. Han, P. Langacker, B. McElrath, and P. Zerwas, Effects of genuine dimension-six Higgs operators, Phys. Rev. D 67, 115001 (2003).

[21] N. Craig, C. Englert, and M. McCullough, New Probe of Naturalness, Phys. Rev. Lett. 111, 121803 (2013).

[22] J. Cohen, S. Bar-Shalom, and G. Eilam, Contact interactions in Higgs-vector boson associated production at the ILC, Phys. Rev. D 94, 035030 (2016).

[23] References are given in J. Tian, presentation at the Asian Linear Collider Workshop 2015, Tsukuba, http://agenda .linearcollider.org/event/6557/. See also the references cited in Appendix A.

[24] T. Ogawa, Study of sensitivity to anomalous HVV couplings at the ILC, at the EPS Conference on High Energy Physics 2017, Venice, https://indico.cern.ch/event/466934/ contributions/2588482/.

[25] Z. Liu, L. T. Wang, and H. Zhang, Exotic decays of the $125 \mathrm{GeV}$ Higgs boson at future $e^{+} e^{-}$lepton colliders, Chin. Phys. C 41, 063102 (2017).

[26] In the description of Higgs physics by dimension-6 operators, the new physics corrections to the hgg couplings actually depend on two EFT coefficients $c_{H t}$ and $c_{G G}$, representing the corrections to the $h g g$ loop diagram from corrections to the $h t \bar{t}$ coupling and from new heavy quarks. These corrections cannot be separated using data from reactions in which the Higgs remains on- shell, so we summarize these effects here in a single parameter called $c_{H g}$.

[27] Determination of $c_{6}$ in this model-independent EFT framework is discussed in [15]. This requires $e^{+} e^{-}$collisions of at least $500 \mathrm{GeV}$.

[28] The strong contribution of future $e^{+} e^{-} \rightarrow W^{+} W^{-}$measurements to global fits to EFT parameters has been emphasized in J. Ellis and T. You, Sensitivities of prospective future $e+e^{-}$colliders to decoupled new physics, J. High Energy Phys. 03 (2016) 089.

[29] I. Marchesini, Report No. DESY-THESIS-2011-044.

[30] A. Rosca, Measurement of the charged triple gauge boson couplings at the ILC, Nucl. Part. Phys. Proc. 273-275, 2226 (2016).

[31] ATLAS Collaboration, Report No. ATL-PHYS-PUB-2014016, 2014.

[32] ATLAS Collaboration, Report No. ATL-PHYS-PUB-2014006, 2014.

[33] J. E. Brau et al. (ILC Parameters Joint Working Group), $500 \mathrm{GeV}$ ILC operating scenarios, arXiv:1506.07830.

[34] The actual program presented in [6] would take only $500 \mathrm{fb}^{-1}$ of data at $380 \mathrm{GeV}$ and then upgrade to a center of mass energy of $1.4 \mathrm{TeV}$. One might question the validity of an EFT analysis at $1.4 \mathrm{TeV}$, and we have not studied this issue sufficiently. We hope that the comparison shown in Table III is sufficiently informative.

[35] The errors estimated in Appendix A for the new physics parameters in $e^{+} e^{-} \rightarrow W^{+} W^{-}$make use of the polarization asymmetry, among other observables. For simplicity, we input the same errors in our fits for unpolarized colliders.

[36] P. Janot, at the FCC Week 2017, indico.cern.ch/event/556692/.

[37] M. Cahill-Rowley, J. Hewett, A. Ismail, and T. Rizzo, Constraints on Higgs properties and SUSY partners in the pMSSM, arXiv:1308.0297.

[38] M. Cahill-Rowley, J. Hewett, A. Ismail, and T. Rizzo, Higgs boson coupling measurements and direct searches as complementary probes of the phenomenological MSSM, Phys. Rev. D 90, 095017 (2014).

[39] S. Kanemura, H. Yokoya, and Y. J. Zheng, Complementarity in direct searches for additional Higgs bosons at the LHC and the International Linear Collider, Nucl. Phys. B886, 524 (2014).

[40] S. Kanemura, M. Kikuchi, K. Sakurai, and K. Yagyu, Gauge invariant one-loop corrections to Higgs boson couplings in nonminimal Higgs models, Phys. Rev. D 96, 035014 (2017).

[41] R. Contino, L. Da Rold, and A. Pomarol, Light custodians in natural composite Higgs models, Phys. Rev. D 75, 055014 (2007).

[42] J. Hubisz, P. Meade, A. Noble, and M. Perelstein, Electroweak precision constraints on the littlest Higgs model with T parity, J. High Energy Phys. 01 (2006) 135.

[43] C. R. Chen, K. Tobe, and C.-P. Yuan, Higgs boson production and decay in little Higgs models with T-parity, Phys. Lett. B 640, 263 (2006).

[44] J. L. Hewett and T. G. Rizzo, Shifts in the properties of the Higgs boson from radion mixing, J. High Energy Phys. 08 (2003) 028.

[45] S. Di Vita, C. Grojean, G. Panico, M. Riembau, and T. Vantalon, A global view on the Higgs self-coupling, J. High Energy Phys. 09 (2017) 069. 
[46] Technically, we convert the $\chi^{2}$ for model A given model B to a $p$-value, then quote this as a number of $\sigma$ s for a 1-degreeof freedom comparison. The result is very close to $\left[\chi^{2}\right]^{1 / 2}$.

[47] J. Yan, S. Watanuki, K. Fujii, A. Ishikawa, D. Jeans, J. Strube, J. Tian, and H. Yamamoto, Measurement of the Higgs boson mass and $e^{+} e^{-} \rightarrow Z H$ cross section using $Z \rightarrow$ $\mu^{+} \mu^{-}$and $Z \rightarrow e^{+} e^{-}$at the ILC, Phys. Rev. D 94, 113002 (2016).

[48] T. Tomita, Hadronic Higgs recoil mass study with ILD at $250 \mathrm{GeV}$, at the Asian Linear Collider Workshop, KEK, Tsukuba, Japan, 2015, http://agenda.linearcollider.org/ event/6557/session/1/contribution/99.

[49] M. Thomson, Model-independent measurement of the $e^{+} e^{-} \rightarrow \mathrm{HZ}$ cross section at a future $e^{+} e^{-}$linear collider using hadronic Z decays, Eur. Phys. J. C 76, 72 (2016).

[50] A. Miyamoto, A measurement of the total cross section of $\sigma_{Z h}$ at a future $e^{+} e^{-}$collider using the hadronic decay mode of $Z$, arXiv:1311.2248.

[51] A. Ishikawa, Search for invisible Higgs decays at the ILC, at the Linear Collider Workshop, Belgrade, Serbia, 2014, http://agenda.linearcollider.org/event/6389/session/0/ contribution/140.

[52] J. Tian, Higgs projections, at the Asian Linear Collider Workshop, KEK, Tsukuba, Japan, 2015, https://agenda .linearcollider.org/event/ 6557/session/12/contribution/129.

[53] H. Ono and A. Miyamoto, A study of measurement precision of the Higgs boson branching ratios at the International Linear Collider, Eur. Phys. J. C 73, 2343 (2013).

[54] F. J. Müller, Report No. DESY-THESIS-2016-018, 2016.

[55] J. Tian, Update of $e^{+} e^{-} \rightarrow \nu \nu h$ analysis, at the ILD Analysis and Software Meeting, 2017, https://agenda.linearcollider .org/event/7703/contributions/39487/attachments/31909/ 48179.

[56] C. Duerig, K. Fujii, J. List, and J. Tian, Model independent determination of $H W W$ coupling and Higgs total width at ILC, arXiv:1403.7734.

[57] H. Ono, Higgs branching fraction study, at the KILC2012 workshop, https://agenda.linearcollider.org/event/5414/ contributions/23402.
[58] L. Liao, Study of $\operatorname{BR}\left(H \rightarrow W W^{*}\right)$ at CEPC, at the 51st General Meeting of ILC Physics Subgroup, 2017, https:// agenda.linearcollider.org/event/7617.

[59] S. i. Kawada, K. Fujii, T. Suehara, T. Takahashi, and T. Tanabe, A study of the measurement precision of the Higgs boson decaying into tau pairs at the ILC, Eur. Phys. J. C 75, 617 (2015).

[60] C. Calancha, Full simulation study on $\mathrm{H} \rightarrow \gamma$ gamma with the ILD detector, at the Linear Collider Workshop 2013, Tokyo, http://agenda.linearcollider.org/event/6000/session/ 31/contribution/180.

[61] H. Aihara, P. Burrows, M. Oreglia et al., SiD letter of intent, arXiv:0911.0006.

[62] C. Calancha, Report No. LC-REP-2013-006, 2013.

[63] M. Pandurovic, Measurement of the $H \rightarrow W W^{*}$ decay at $500 \mathrm{GeV}$ ILD and at $3 \mathrm{TeV}$ CLIC, at the International Workshop on Linear Colliders (LCWS16), Morioka, 2016, https://agenda.linearcollider.org/event/7371/contributions/ 37896/.

[64] R. Karl, Prospects for electroweak precision measurements and triple gauge couplings at a staged ILC, at the EPS Conference on High Energy Physics 2017, Venice, https:// indico.cern.ch/event/466934/contributions/2589875/.

[65] C. Patrignani et al. (Particle Data Group), Review of particle physics, Chin. Phys. C 40, 100001 (2016).

[66] A. Kotwal et al., Working Group Report: Precision study of electroweak interactions, in Proceedings of the APS DPF Community Summer Study (Snowmass 2013), edited by N. Graf, J. L. Rosner, and M.E. Peskin, arXiv:1310.6708, http://www.slac.stanford.edu/econf/C1307292/.

[67] S. Schael et al. (ALEPH, DELPHI, L3, OPAL, and SLD Collaborations, LEP Electroweak Working Group, SLD Electroweak Group, and SLD Heavy Flavour Group), Precision electroweak measurements on the $Z$ resonance, Phys. Rep. 427, 257 (2006).

[68] See Supplemental Material at http://link.aps.org/ supplemental/10.1103/PhysRevD.97.053003 for the linear relations used in our fits and for the final output covariance matrices. 